\title{
Undergraduate Engineering Research Day 2020
}

The University of Toronto's Undergraduate Engineering Research Day (UnERD) is an annual conference aimed at providing an opportunity for undergraduate engineering students to showcase their research to industry professionals and fellow students, inspiring the exchange of innovative solutions across a wide breadth of global challenges.

STEM Fellowship came together with the UnERD organizers to provide a unique opportunity to the engineering students to publish their work in our STEM Fellowship Journal. For all the variation between project themes, it remains that all submissions are of incredibly high quality. Every abstract is demonstrative of immense creativity and high potential on the respective team's part.

On behalf of STEM Fellowship, I would like to extend my heartfelt congratulations to all students who participated in UnERD, and I wish them all the best for their future endeavours in research and engineering. It has been a privilege for us to witness the research capabilities of the next generation of students firsthand, and I am certain all entrants will continue to demonstrate excellence in their respective research careers.

\section{Harsukh Benipal}

Associate Science Communication Chief, STEM Fellowship

Managing Editor, STEM Fellowship Journal

\section{Dr. Sacha Noukhovitch}

Founder, President and Editor-in-Chief

STEM Fellowship/STEM Fellowship Journal

\section{Disclaimer}

These abstracts are provided for all student teams that have submitted to UnERD 2020. The STEM Fellowship Journal editorial

board has made every effort to ensure proof and English editing of these abstracts in a limited amount of time, and neither

organization as a whole or any of its volunteer members can be held accountable for inaccuracies that may have occurred in the abstract publication. Abstracts are published in alphabetical order per school names of the participating teams. 
Hardware Implementation of a Spike Classifier for Real-time Closed-loop Neural Interfaces

\section{Ahmed Abdelmoneim, Gerard O'Leary, Roman Genov}

Edward S. Rogers Sr. Department of Electrical and Computer Engineering, University of Toronto; Institute of Biomedical Engineering, University of Toronto

The activation of a neuron results in electrical signatures known as action potentials. These spikes can be recorded in vitro using microelectrode arrays (MEAs) to investigate the mechanisms which give rise to the brain's emergent behavior and consciousness [1]. Each 30um electrode in an MEA captures the activity of up to dozens of neurons within a radius of $100 \mathrm{um}$. Spike classification is the process of matching detected spikes with the exact neurons that generated them. For neural plasticity experiments involving closed loop stimulation of neurons, this classification must be carried out in real-time with a latency on the order of milliseconds [2].

The purpose of this project is to investigate the effectiveness of a Machine Learning based spike classifier. This classifier is trained using data on pre-recorded spikes and their known neuron sources to extrapolate patterns to new incoming spikes. A software benchmark has been developed to measure accuracy through the number of falsely classified spikes in a test recording, achieving a score of $94 \%$. Next, this classifier will be implemented on a field programmable gate array (FPGA) to exploit parallel computation and reduce latency. Resource usage, measured by the number of utilized FPGA look-up tables and registers, is to be minimized to allow for scalability. Different hardware optimizations will be explored to achieve a balance between latency and resource usage. This implementation will then be compared against existing classifiers $[3,4]$ using the aforementioned metrics. With low latency, this classifier will aid in the study of complex neuron populations.

Computational Protein Design - A Gaussian Process Regressor Based Machine Learning Model For Phosphatase Activity Prediction Anaqi Afendi, Paul Xu, Krishna Mahadevan
Department of Chemical Engineering and Applied Chemistry, University of Toronto; Biozone - Centre for Applied Bioscience and Bioengineering, University of Toronto

Current state of the art methods of lab testing new proteins, even in vitro, is extremely cost, labour and time intensive and for shorter proteins has a search space of up to $10^{\wedge} 130$ ! It is therefore important that when we do carry out these experiments to find new/better proteins, we are able to narrow down our search space to the most promising regions. Analyzing protein sequences manually is a very long and tedious process, and there are pitfalls that we can have as humans. Mainly, we assume that we can use encoding and characterizing methods that we are familiar with, even though they might not necessarily be the best/actual features that proteins express to produce their results.

My project has focused on the computational design of phosphatase proteins [1]. We developed machine learning models which when given a protein sequence input, was designed to predict what the protein activity would be on a given substrate. I will be presenting on a machine learning pipeline model we made that utilizes one-hot encoding to convert protein sequences data into numerical data, followed by a gaussian process regressor to perform modelling [2][3]. This model was trained on a set of protein sequences from the Haloalkanoic Acid Dehalogenase (HAD) superfamily of proteins and tested on different sequences from the same distribution. This gaussian process regressor was able to accurately predict protein activity of phosphatases on some substrates, with an average prediction to true value correlation coefficient of $\mathrm{R}^{\wedge} 2=0.7$ in some cases. Researchers can feed in protein sequences to these models and get a prediction of what the enzyme activity would be like. They can help researchers narrow down their search space to search in regions providing only the most promising protein sequences.

Development of an Al-Assisted Dynamic Quiz System for Student Assessments

\section{Brohath Amrithraj, Ariel Chan}

Department of Chemical Engineering and Applied Chemistry, University of Toronto; Institute for Studies in Transdisciplinary Engineering Education and Practice (ISTEP) 
Assessments such as exams, essays, and discussions are employed to determine the effectiveness of learning. Exams involving multiple-choice questions, true or false, and short answers are used for their rapid grading and timely feedback to students. However, these questions are generally the same amongst students and do not account for their skill level. Recent advances in artificial intelligence ( $\mathrm{Al}$ ) research has enabled its application in education and has focused on automating administrative tasks, intelligent tutoring systems, personalizing feedback, and predicting student performance [1], [2].

We aim to improve personalized learning by using Alalgorithms to create a dynamic quiz system that has an embedded feedback algorithm to adjust question difficulty, appropriately challenge students, and identify their weaknesses. To accomplish this, the development of a deep neural network that can adapt to a student's learning level is explored.

The algorithm is expected to assess students' level using their previous academic performance and assign an appropriate question. Once their answer is evaluated, the algorithm will re-evaluate the student's level, and assign a new question based on updated student data. The cycle will continue until a predetermined stop condition is reached. If the algorithm can predict students' responses at an accuracy of at least $80 \%$, this prediction can be used to assign personalized questions in assessments. Currently, the algorithm is being coded, and the next step is to train it with student data and test it in a course. The improvement of the algorithm is iterative and will focus on improving quality of questions assigned.

\section{Best practices for effective teamwork in online} first-year design courses

\section{Julianne Attai, Katherine Whelan, and Patricia Sheridan}

Division of Engineering Science; Troost Institute for Leadership Education in Engineering; Institute for Studies in Transdisciplinary Engineering Education and Practice, University of Toronto

Effective communication and collaboration in multidisciplinary teams are typically a key learning outcome in engineering cornerstone courses. Social distancing and the rise of the coronavirus has forced many teams to become fully virtual, raising the importance of designing virtual environments and identifying best practices for effective remote teamwork.

We define an effective team as being able to understand and build on each other's ideas, and communicate effectively and resolve conflicts [1]. This formed the framework for our research. To better understand the many factors that influence team effectiveness, we did a broad literature review on remote teams and engaged with faculty members and students about their online transition this semester. We have identified recurring points of contention relevant to the remote teamwork experience.

We are currently assembling a handbook discussing how different factors influence team effectiveness, with a focus on the implications of remote collaboration. Specific topics discussed in the handbook include: trust and accountability in remote teams, intersection of identity online, effective communication patterns, affordances of technology in collaborative work, and anticipated challenges. We are also recommending best practices on how each of these factors can be leveraged in remote environments to promote effective teamwork.

The handbook will be adaptable to different teams' needs, explicit but not prescriptive, and appropriate for the firstyear team experience. While we've scoped our research to consider primarily the first-year collaborative experience in cornerstone courses (i.e. ESP and Praxis), this handbook can be utilized to generally enhance collaborative learning and teamwork online.

Rationally designed hydrolase variants for the degradation of polyethylene terephthalate Joseph Bellissimo, Radhakrishnan Mahadevan

Department of Chemical Engineering and Applied Chemistry, University of Toronto; iGEM Toronto, University of Toronto; Institute of Biomaterials and Biomedical Engineering, University of Toronto

359 million tonnes of plastic were produced globally in 2018 [1], which was nearly 100 million tonnes higher than the per annum production a decade earlier [2]. Polyethylene terephthalate (PET), commonly used in packaging and textiles, constitutes $64 \%$ of global synthetic fibre production [3] and is 
extremely resistant to environmental degradation [4]. Current mechanical and thermochemical recycling processes for PET are limited by the loss of favourable mechanical properties, especially decreasing intrinsic viscosity values below 0.70 [3], [4]. Enzymatic hydrolysis of PET has been proposed as a possible solution for $\mathrm{PET}$ recycling as it maintains the intrinsic viscosity of the recycled PET and is sustainable. However, all of the naturally identified PET hydrolases have low stability at the glass transition temperature of $70^{\circ} \mathrm{C}$ and catalytic activity below 700g PET L-1 h-1 which is required to be cost competitive [5]. Recently, several engineering attempts have been made on the PET hydrolyzing enzymes PETase and LCC which improved the melting temperature to $94^{\circ} \mathrm{C}$ and catalytic activity to 19.35g PET L-1 h -1 [4], [5].

Here we build on current progress using a rational design approach to optimize the catalytic activity and thermostability of the PETase, LCC and MHETase enzymes to the required levels for industrial integration. We designed disulfide bonds, improved hydrophobic packing and identified stabilizing single mutations using quantum physical models [6]. We combined these single mutants to generate high performing multi-mutant enzyme variants with minimized antagonistic epistatic effects [7]. We will validate their catalytic activity, thermostability and folded configurations using molecular dynamics simulations to determine whether these variants are industrially competitive. Lastly, the successful variants will be integrated into a stirred tank bioreactor to convert PET into its monomers ethylene glycol and terephthalic acid to complete the circular economy.

\section{Role Play-Based Training: Developing Confidence and Empathy for Engineering Instructors}

Audrey Benmergui, Cori Hanson, Deborah Tihanyi

Institute for Studies in Transdisciplinary Engineering

Education \& Practice (ISTEP), University of Toronto; Vice Dean Undergraduate Office, Faculty of Applied Science and Engineering, University of Toronto; Leadership, Adult \& Higher Education, Ontario Institute for Studies in Education, University of Toronto; Engineering Communication Program (ECP), University of Toronto

An unexpected challenge course instructors face is discussing sensitive topics with students. Instructors must respond quickly to unexpected and unpredictable remarks while unrecognized implicit biases may impact the effectiveness of the conversation [1]. Navigating these issues requires empathy, preparedness, and bias recognition.

These skills, however, require practice and experience. Role plays and simulations have been used in various fields (medicine [2], aviation [3], counselling [4], and more) for gaining practice and experience to develop participants' improvisational and empathetic skills [5], without the risks inherent in a real-life situation. However, there is a lack of such role-play scenarios addressing the challenges that are unique to engineering instructors.

This project aims to develop scenarios, which simulate difficult discussions specific to engineering education, that instructors can use as a training tool to practice empathetic and improvisational skills in a low-risk environment and facilitate discovery and reflection on internalized biases. To do so, we have developed an interactive scenario that addresses the complex issues involving academic integrity in a remote learning environment.

For the scenario to be effective, it should be realistic, engaging, and promote self-reflection. Realism and engagement of the scenario are gauged by observations of participants during the role-play activity as well as postscenario feedback. Self-reflection is ensured via debriefs throughout and after the scenario. The success of the scenario in increasing participants' empathy and preparedness for difficult discussions is measured by standardized pre- and post-scenario surveys.

Based on results, the scenarios will be iterated to increase their effectiveness and success. We expect scenarios to heighten the participants' empathy towards their students and improve their ability to manage sensitive topics with increased confidence, ultimately improving the learning experience for educators and students.

\section{Volitional Control of Single Neurons in the Rodent Hippocampus}

Michael De Biasio, Kramay Patel, Taufik Valiante

Institute of Biomedical Engineering, University of Toronto; Krembil Research Institute, Toronto Western Hospital (TWH); Faculty of Medicine, University of Toronto; Division of Neurosurgery, Department of Surgery, University of Toronto; 
The KITE Research Institute, University Health Network; Electrical and Computer Engineering, University of Toronto;

The brain is composed of billions of interconnected neurons. Recent studies have shown that animals can learn to control individual neurons in neocortical regions, facilitated by brain networks that govern skill learning in our day-today lives [1]. We recently demonstrated in humans that this learning can be achieved in paleo-cortical regions such as the hippocampus, the primary brain structure for memory [2]. However, since the paleocortex does not participate in the same skill-learning networks as the neocortex, we wanted to investigate the brain networks involved in this type of hippocampal skill learning. To do this, we are implanting Long Evans rats with microelectrode arrays targeting multiple brain regions to extract single neuron activity. The single neuron activity is used in an auditory feedback task to train animals to control individual hippocampal neurons via operant conditioning. As the animals learn the task, we are probing the underlying neural networks to understand which networks are involved in this type of learning. Thus far, we have completed testing on two pilot animals. We hypothesize that (1) rats, much like humans, will learn to control activity of individual hippocampal neurons, and (2) the ventral striatum, a brain region that bridges the motor and limbic systems, plays a key role in facilitating this type of skill learning. A better understanding of the interactions between the striatum and hippocampus may be instrumental for furthering our understanding of the interactions between various memory systems. This understanding can inform novel ways to improve memory function in patients with dementia.

\section{Bridging the Divide: Engineering the Tumour Microenvironment}

\section{Eryn Bugbee, Natalie Landon-Brace, Alison P.} McGuigan

Institute of Biomaterials and Biomedical Engineering, University of Toronto; Chemical Engineering \& Applied Chemistry, University of Toronto

Immunotherapy has become a mainstay in modern cancer treatment regimens. Despite impressive success in some diseases, one of immunotherapy's critical shortcomings is its inability to overcome the barriers of the tumour microenvironment (TME) [1]. The TME is a complex entity that encompasses a heterogeneous set of components including circulatory networks, immune cells, fibroblasts, and the extracellular matrix (ECM) [2]. Enhanced understanding of the underlying mechanisms governing tumour-immune interactions in the context of the TME is essential to improving the efficacy of immunotherapy.

The purpose of this review is to discuss the major facets of the TME and comprehensively examine 3D in vitro models to probe the effects of these features. A series of search strings were used to explore multiple databases including PubMed, Wiley Online Library, and Scopus; certain highimpact journals were also searched independently. Selected literature was screened for relevance and compiled into an inclusive literature review.

The identified TME factors were broadly categorized as follows: immunogenicity, tumour architecture, metabolism, and hypoxia. In parallel, a summary of in vitro tumour models was created for each category. This summary includes the tissue roll for analysis of cellular environment and response (TRACER), a paper-stacked model developed by the McGuigan Lab recapitulating the oxygen and metabolites gradients present in the TME [3]. Future work will implement this information to explore TME-immune interactions suitable for modelling in TRACER. Overall, the goal of this work is to facilitate the integration of biological and engineering knowledge, in hopes of elucidating mechanisms to enhance the success of cancer immunotherapy.

\section{Detecting Covid-19 in Chest X-Rays using Transfer Learning}

\section{Amy Chen, Jonathan Jaegerman, Professor Jonathan Chan}

\section{University of Toronto; King Mongkut's University of Technology Thonburi}

The recent Covid-19 outbreak has impacted countries worldwide and there is a need for quick and accurate screening methods. The main testing method involves using reverse-transcription polymerase chain reaction which can be time-consuming and laborious [1]. Chest X-Ray scans are a more widely available and cheaper alternative. However, as hospitals become overloaded, there is an increasing need 
for computer-aided methods to improve the efficiency of diagnosis [2]. Many publications suggest machine learning models that use transfer learning and build on pre-trained models, including VGG, Inception, and ResNet, to detect Covid-19 [3][4] but have not incorporated the newly available X-Ray image data.

The purpose of this project is to design a machine learning model that integrates new image data and transfer learning to automatically detect Covid-19 from Chest X-Rays. The model should be accurate and have a low false-negative score to prevent misdiagnosis. The model should also be designed to prevent overfitting and allow for multiclass classification and interpretable results. Multiple performance metrics including the accuracy and f-score will be used to quantitatively evaluate the model.

Several machine learning models have been built from existing models with pre-trained weights. Updated and publicly available X-Ray images have been preprocessed and split into training, validation, and testing sets. After the models are trained and tested with the data, the performance metrics will be used to compare their effectiveness in diagnosing Covid-19. Further refinements will be made to decrease overfitting and increase model interpretability to allow for a more robust Covid-19 detection method.

\section{Single Resource Model for Parallel Machine Job Shop Scheduling Using Constraint Programming} Yihan (Cathlyn) Chen, Litong Zheng, Kyle Booth, Christopher Beck

\section{Department of Mechanical and Industrial Engineering, University of Toronto}

The job-shop problem (JSP) is a classical scheduling process in many manufacturing and service industries that assigns multiple jobs on several processing machines [1]. We investigate an instance of JSP involving identical parallel machines and sequence-dependent setup times, with the objective to minimize the makespan [2]. Previous works have presented numerous mathematical techniques, including constraint programming (CP) with alternative resources [3]. However, recent work has developed a single resource CP model with proven superiority to the existing alternative resource model in vehicle routing problems (VRP), which is mathematically similar to JSP [4]. Our work applies the same single resource model in JSP using one machine on an extended time horizon. We hypothesize it to demonstrate similar advantages as in VRP with higher runtime efficiency and reduced memory usage.

We implement both models in software starting with the high-level CP solver MiniZinc. We run experiments with each model solving benchmark instances, then analyze and compare the numerical results. We use the CP Optimizer for further investigation, as MiniZinc lacks control over low-level details. Our results show the single resource model solved by CP Optimizer finds better objective values with less memory usage. Since the complexity of JSP is categorized as NPhard, there is ongoing research proposing new optimization methods to tackle it [5]. This model improves both space and time efficiency and reduces computational costs. We also find the advantages exhibited in VRP are more prominent, so we conclude this model can be beneficial for other routing problems in future work.

\section{Mitigating systemic risks by Quantum Annealing}

\section{Cheng Chi,Amine Aboussalah, Chi Guhn Lee}

Institute of Industrial and Mechanical Engineering, University of Toronto

Modern financial system witnesses the increase of interdependencies among organizations such as banks, companies or even countries. One such financial system is interbank system where banks connected by their interdependencies such as interbank loans form the interbank network. Such interdependencies can amplify financial shocks, spread the financial contagions and breeds cascade failures in the network. How to mitigate such cascade failures has been a popular research topic. For instance, mitigating cascade failures is approximated by a Mixed Integer Linear Programming problem(MILP) with interdependencies being decision variables [1]. This classical algorithm will return the optimal network structure that can incur least cascade failures. However, this MILP is a large-scale optimization problem and can be intractable when the size of network is large. In our research, we will implement Quantum Annealing algorithm in D-wave quantum annealer to solve this large scale optimization problem, during this process we will modify the MILP so that it can be mapped to the quantum 
annealer device. After having a returned optimal solution corresponds to the optimal interbank network structure, we will implement a recursive simulation algorithm to test whether the cascade failure is mitigated or not. During the simulation, we will perturb the network with financial shocks, penalize the banks that fail during the financial contagion with discontinuous value loss, and calculate the new bank value recursively to capture new failures. During this process, bank failures will come in waves and the simulation will terminate only when no new failures occur. We will run simulation for both the non-optimal interbank network and optimal network obtained from quantum annealer, and if there is a significant decrease in number of total failures for optimal network, the cascade failures are mitigated. The main contribution of our research would be using quantum annealing to make the search for the optimal solution for current Mixed Integer Linear Programming tractable when the number of decision variables is large in a interbank network with many banks. And we will also test the effect of this optimization on mitigating cascade failures in a relatively realistic simulation with discontinuous value loss for banks and recursive calculations of bank values.

\section{Ontology Optimization Tools for Quick and Meaningful Communication}

\section{Amanda Chow, Michael Gruninger}

Department of Industrial Engineering, University of Toronto

The Common Logic Repository (COLORE) is the largest testbed of ontologies in the world [1]. As artificial intelligence undertakes increasingly important roles, the computer depends on ontologies to understand meanings and relationships of concepts in different subject areas. To better understand an ontology, consider the theory "betweenness". What rules must be satisfied for something to be "between" other things? These "rules" are called axioms. Then, there are other theories in the ontology that may entail "betweenness", such as "linearity". Does "linearity" convey everything deduced from "betweenness"? What about the other way around? Or are they independent of one another? With two theories, their relationship can be determined by manually entering axioms into a theorem prover. However, COLORE currently has 2580 ontologies (each with many theories!) [2].

The motivation of this project is to support the growth of
COLORE as new theories are added. Since a manual process is timely and prone to error, the gap is a program that identifies if and where a theory belongs in an ontology. The process should accurately identify the position and only require one manual input of the new theory.

Currently, one program has been implemented to find the relationship between two theories. Another program uses this to insert a new theory into the existing linear chains. The next step is to include a search function that locates equivalent theories, which has many useful applications. With more tools that optimize user interactions, the more readily computers can use this information to make intelligent decisions.

\section{Automated Construction and Visualization of Bibliometric networks for Understanding Research Collaborations}

\section{Kevin Da, Henrik Persson, Noah Frank, Craig Simmons}

Translational Biology and Engineering Program, Ted Rogers Centre for Heart Research, University of Toronto

Interdisciplinary research collaborations can help advance productivity, drive innovation, and foster connections within academic networks. Measuring this impact quantitatively through a network analysis of bibliometric data helps provide statistical evidence showing how collaborations change over time. Popular analytical tools such as Gephi [1] and VOSviewer [2] can be used to construct visualizations and generate statistics of these co-authorship networks. However, these tools are prone to error as users must manually extract publication information and offer little flexibility in filtering a network. An alternative open-source software offering more flexibility and dynamic network visualizations is Kumu, but this program requires specifically formatted files.

An easy-to-use software program has been designed to automate the construction and analysis of bibliometric networks while generating Kumu-compatible outputs. A Scopus RESTful API wrapper will be used to extract publication information from the Scopus database and organized into bibliometric networks using a custom Python program. After establishing a workflow, bibliometric networks will be constructed for interdisciplinary programs such as the Translational Biology and Engineering Program across 
different time spans. To verify accuracy and reproducibility, these networks will be compared to previously constructed networks using other statistical software with network metrics such as centrality and betweenness [3]. This process will allow users to construct accurate networks in an automated fashion and have the flexibility of working with Kumu. A functional, user-friendly interface has been developed to facilitate widespread adoption of the program.

\section{Exploring Applications of Collaborative Multi- User Computer-Aided Design Software on the Future of Engineering Education}

\section{Yuanzhe Deng, Alison Olechowski}

Department of Mechanical and Industrial Engineering, University of Toronto; Institute for Studies in

Transdisciplinary Engineering Education and Practice, University of Toronto

As multi-user computer-aided design (CAD) software launches for the market in recent years, it aims to build a collaboration platform for simultaneous CAD modelling between geographically scattered engineers. This unique characteristic has drawn attention to its potential of improving the efficiency of engineering design and possible applications on engineering education. However, insufficient research has been conducted in the field of collaborative learning with cloud computing tools, especially on CAD software specifically [1]. This research examines the feasibility of applying the collaborative multi-user CAD software on engineering education by grouping students with diverse CAD experience for an open challenge.

Currently, a trial experiment has been conducted on Onshape, a commercially available multi-user CAD software, which records real-time editing history by every editor in the program. These backend analytic data are reformulated to reveal the performance of the team. With the additional design of a post-study survey, responses can be analyzed through qualitative coding to identify remarkable advantages of such learning methods and students' dissatisfactory experience.

Combining analysis of analytic data and survey responses, it is hypothesized that educators can identify the optimal teaching method, benefiting students' learning experience, through the use of multi-user CAD. Applying this collaborative platform, traditional engineering education on CAD modelling will be augmented by providing cognitive apprenticeship training, which has been shown being able to lead students to more successful problem-solving [2]. In the future, actual experimental data will be analyzed using this model to discover a better collaborative teaching approach for engineering students on CAD.

Validation of an image analysis algorithm for 3D hydrogel-culture platform GLAnCE

Kirtana Devaraj, Subaita Rahman, Divy Raval, Michael Paraskevopoulos, Nila Wu, Alison McGuigan

Institute of Biomaterials and Biomedical Engineering University of Toronto; Department of Chemical Engineering and Applied Chemistry

GLAnCE (Gels for Live Analysis of Compartmentalized Environments) is a 3D hydrogel-culture platform within a 96-well plate format developed by the McGuigan Lab. GLAnCE's unique seeding method generates consistent thin hydrogel layers that are advantageous for image acquisition using a standard widefield microscope, with the option to continuously visualize cultures in real-time. This image-based approach allows for the observation of cell-cell interactions to probe tumour microenvironment questions posed at the in vitro cancer drug discovery stage [1].

The gap is that analysis of acquired images is time consuming, as it requires manually selecting a region of interest (ROI) per well of the plate to access the output value. The objective of this project is to develop and validate an automated image analysis pipeline to facilitate analysis of images acquired with GLAnCE. The developed Python-based algorithm will automatically detect a ROI per well while eliminating any artifacts and areas of low/high cell density.

The validation process ensures that the algorithm produces accurate results with less subjectivity than the manual process. Mean gray values and presence of artifacts in the ROIs obtained with both approaches will be compared. These metrics help identify the difference between a cell, the channel and low/high concentration of cells. The efficiency of the algorithm will be determined using graphical analysis, allowing for the algorithm to complement the device. Future studies include modifying the algorithm for more advanced 
image analysis purposes such as the presence of multiple fluorescent channels and accessing 3D information from widefield images.

\section{Functional maturation of human pluripotent stem cell-derived cardiomyocytes in 3D tissue constructs using chemically-defined maturation media}

\section{Lauren Durland, Neal Callaghan, Craig Simmons}

Institute of Biomaterials and Biomedical Engineering, University of Toronto; Department of Molecular Genetics, University of Toronto; Translational Biology and Engineering Program, Ted Rogers Centre for Heart Research; Department of Mechanical and Industrial Engineering, University of Toronto

ardiovascular disease is the leading cause of death worldwide [1], while cardiotoxicity is the single most common reason for pharmaceutical clinical trial failure or postrelease recall [2]. Nevertheless, existing preclinical models of the myocardium are poorly predictive or representative of cardiotoxicity and pathological features [2]. The use of cardiomyocytes from induced pluripotent stem cells (iPSC$\mathrm{CMs}$ ) in 3D myocardial co-culture may provide an alternative to these models; however, these engineered tissues are still immature in terms of morphology and functional metrics, including electrophysiology, calcium handling and force generation [3]. The culture media most commonly used in these tissues are xenogeneic or otherwise adapted from neuronal culture media, and therefore do not represent the biochemical niche of the developing myocardium. This project aims to demonstrate that novel, chemicallydefined media formulations can enhance the maturity of iPSC-CMs in in the Biowire II 3D tissue construct, relative to gold-standard commercial or neuronal media. While the components of these media would be expected to have relatively small influences individually, we expect that these factors in combination will act synergistically to induce a level of maturation comparable to those observed in existing 3D models, and in primary adult CMs. Morphological maturity will be assessed by immunohistochemical detection of adult-like structural features, including uniaxially aligned sarcomeres, T-tubules and gap junctions [4]. Concurrently, calcium and voltage imaging and contractile force will be used to assess functional maturity [3]. Validated, chemically-defined iPSCCM medium will provide a tool for diverse applications, including cardiotoxicity panels, drug efficacy studies, and disease modeling.

Impact of Multi-Bounce Light Propagation on Fluorescence Imaging for Oral Cancer Surgery Jacqueline A. Fleisig, Johnathan C. Irish, Michael J. Daly

Institute of Biomaterials and Biomedical Engineering, University of Toronto; TECHNA Institute, University Health Network; Otolaryngology-Head \& Neck Surgery, University of Toronto; Princess Margaret Cancer Centre, University Health Network, Toronto, Canada

Novel tumour-targeting fluorescent contrast agents for oral cancer combined with improved in-vivo imaging systems have the potential to increase surgical precision and improve patient outcomes [1]. For advanced fluorescence imaging techniques, understanding how light propagates within the structure of interest (here, the oral cavity) is essential [2]. Lighting models which account for the relative position of the lighting source from the surface ('direct lighting') are well-documented [3]. However, in the oral cavity where the surface is nearly spherical, multi-bounce light reflection and propagation ('global lighting') may also have a significant impact on light distribution. Here, a computational model was developed to simulate global lighting effects and determine their impact on light propagation within the oral cavity. To account for global lighting, after computing direct lighting intensity, light is added with a series of reflective 'bounces'. In a given 'bounce', light from each origin face on the surface is multiplied by an attenuation parameter and added to each recipient face. Simulations were validated by comparing to 30 endoscopic images of an agar oral cavity phantom. Global lighting simulations increased maximum light intensity by $50 \%$ on average and showed greater agreement with endoscopic images than simulations that used only direct lighting, with the average pixel-to-pixel ratio between the endoscopic image and the simulation image decreasing from 1.61 (std: 1.22) to 0.86 (std: 0.24 ). Thus, for light models in the oral cavity, global lighting plays a significant role in light propagation and accounting for it provides more accurate modelling. Next steps include integrating this model into 
fluorescence imaging systems currently in development, which requires improved software and further validation on phantoms and in-vivo models.

\section{Effect of Astrocyte-to-Neuron Reprogramming in the Medial Prefrontal Cortex (MPFC) after Ischemic Stroke}

\section{Donna Gao, Tom Enbar, Maryam Faiz, Cindi Morshead}

Institute of Biomaterials and Biomedical Engineering, University of Toronto; Department of Surgery, Division of Anatomy, University of Toronto; Institute of Medical Science, University of Toronto; Donnelly Centre for Cellular and Biomolecular Research, University of Toronto

Stroke in one of the leading causes of adult disability, with many survivors suffering cognitive impairments. There are no current treatments, outside of rehabilitation, that significantly improve stroke outcomes [1]. The need for novel therapeutics is evident.

One potential strategy is direct cellular reprogramming. We propose using the transcription factor NeuroD1, which has been shown by Guo et al. to effectively convert astrocytes to neurons in vitro, to generate new neurons that replace those lost in stroke [2]. We hypothesize that expression of NeuroD1 in astrocytes will result in their conversion to mature neurons, reversing cognitive deficits caused by stroke in the medial prefrontal cortex (MPFC).

To accomplish this, an adeno-associated virus (AAV5) delivered NeuroD1 to glial fibrillary acidic protein (GFAP) expressing cells, a known marker of astrocytes [3]. Creconditional reporter mice were used to track AAV5 infected cells, and were injected with either AAV5-Cre (control) or AAV5-Cre-NeuroD1. The percentage of reprogrammed neurons (expressing NeuN) over total transfected cells was then assessed.

As predicted, at early times post-AAV5 transfection, the majority of infected cells were GFAP expressing astrocytes. However, at longer times, both the control and experimental group reported similar numbers of reprogrammed neurons. These unexpected findings suggest that MPFC neurons express low levels of GFAP, a prospect currently being examined using RNA-scope. With regards to behavioural outcomes, a battery of behaviours assays revealed that
NeuroD1 expression rescued cognitive impairments in this stroke model. Our findings thus far support the hypothesis that reprogramming is a potential therapeutic to promote stroke recovery.

\section{Affinity Controlled Release of Rod-derived Cone Viability Factor from an Injectable Hydrogel Drug Delivery Vehicle}

\section{Bipasha Goyal, Carter J. Teal, Hidekiyo Harad, Thierry Léveillard, Philippe Monnie, Molly S. Shoichet}

Division of Engineering Science, University of Toronto; Institute of Biomaterials and Biomedical Engineering, University of Toronto; Department of Chemical Engineering and Applied Chemistry, University of Toronto; Vision Division, Krembil Research Institute, Krembil Discovery Tower; Department of Physiology, Faculty of Medicine, University of Toronto; Department of Genetics, Institut de la vision, Inserm, Université Pierre et Marie Curie

Retinitis pigmentosa (RP) is a genetic disease that causes rod photoreceptor cell degeneration, subsequently leading to cone photoreceptor cell loss and eventual blindness. Rodderived cone viability factor (RdCVF), a potential therapy for $R P$, is a paracrine factor secreted by rod photoreceptors that maintains the structure and function of cone photoreceptors. However, administration of protein therapeutics to treat retinal degenerative diseases remains challenging. Topical applications are limited by rapid drug clearance and bolus injections by risk of overdose. To mitigate these risks, we developed an affinity-based hydrogel drug delivery vehicle for intraocular protein delivery. The hydrogel consists of an injectable physical blend of hyaluronan and methylcellulose (HAMC) modified with a peptide binding partner of the Src homology 3 (SH3) domain that interacts with the RdCVF-SH3 fusion protein, enabling its controlled release.

To develop this protein delivery system, RdCVF-SH3 was first expressed and purified from E. coli. Purification products were analyzed with mass spectrometry and SDS-PAGE. To assess bioactivity, chick retinal explants were harvested and treated with the recombinant protein. Cone cell viability was quantified after 7 days. Treated explants showed a two-fold increase in cone cells relative to a negative control. Once the efficacy of the protein was understood, the release profile of 
RdCVF-SH3 from HAMC-binding peptide compared to simple HAMC hydrogels was investigated. Using a custom ELISA, it was demonstrated that the peptide modified hydrogel sustained release for 7 days.

The controlled release of RdCVF-SH3 thus has the potential to increase viability of cone photoreceptor cells in vivo. Moreover, our affinity-based system constitutes a versatile intraocular delivery platform for the treatment of retinal degenerative diseases.

Teaching Teamwork, Ethics and Equity, and Impact on Society to Undergraduate Engineering Students: A Review of the Literature

\section{Ellie Grushcow, and Patricia Sheridan}

Division of Engineering Science, Troost Institute for Leadership Education in Engineering, Institute for Studies in Transdisciplinary Engineering Education and Practice

Since 2015, the accreditation of Canadian engineering programs has centered on twelve 'graduate attributes,'[1] overarching groups of characteristics that graduates of accredited programs should develop. These attributes are derived from the Washington Accord, an international agreement between countries ensuring that qualifications from their respective engineering schools are "comparable" and "substantially equivalent."[2] Despite the importance of these attributes in the accreditation process, there has been relatively little discussion of the trends in teaching each attribute or of teaching certain combinations of attributes together.

This work seeks to understand current practices and trends in teaching combinations of the attributes "(Individual and) Team Work," "Ethics and Equity," and "Impact of Engineering on Society and the Environment." We chose these attributes because we believe that teaching these attributes together constitutes a large part of the teaching of good engineering leadership, a priority of Troost ILead.

Conducting a literature review following systematic principles, papers will be gathered using replicable searches of a wide variety of databases. Papers will be selected based on predetermined inclusion and exclusion criteria such as the date of publication and the explicit instruction of at least two attributes as part of the intervention. Data will be collected from each paper, including the motivation for each intervention and what pedagogy is being used. This data collection process is currently underway.

From this review, we expect to gain a clear understanding of where, why, and how these attributes are being taught together in undergraduate engineering classrooms in a variety of Washington Accord countries. Understanding what others are already doing will allow us to better inform how we teach these attributes together at the University of Toronto. We also hope that this work will be helpful to other educators seeking to teach these or similar attributes at their own institutions.

\section{Effect of Extracellular Matrix and Signaling Proteins on Self-assembly of Liver Biliary Cells in Hyaluronan Hydrogels}

\section{Chengyu Guo, Muhammad Rizwan, Molly Shoichet}

Institute of Biomaterials and Biomedical Engineering, University of Toronto; Department of Chemical Engineering and Applied Sciences, University of Toronto; Department of Chemistry, University of Toronto

There is a critical need to develop liver organoids for drug discovery and transplantation. However, the current liver organoids developed in vitro do not have bile ducts, which is critical to long-term liver function, and they are unsuitable for transplantation due to the use of mouse tumor-derived biomaterials [1]. Jagged1-induced Notch signaling [2], transforming growth factor beta 1 (TGF $\beta 1$ ) [3], and composition of the 3D microenvironment [4], are known to modulate self-assembly of cholangiocytes (liver biliary cells) to form liver biliary tree. However, the effect of jagged1 and TGF $\beta 1$ on cholangiocyte growth and self-assembly in 3D hydrogel microenvironment is unknown. We hypothesize that the 3D culture of mouse cholangiocytes in hyaluronan (HA; an essential component of liver) hydrogels, in the presence of TGF $\beta 1$ and/or jagged1, will improve growth and cellular selfassembly of cholangiocytes. We cultured cholangiocytes in HA based hydrogel, crosslinked with oxime click chemistry, and studied the effect of signaling proteins and gel composition on cell growth and self-assembly using PrestoBlue ${ }^{\mathrm{TM}}$ assay 
and image analysis. Inclusion of laminin or collagen in the HA gels improved growth rate of cholangiocytes considerably. HA alone did not induce 3D cell spreading. Jag1 did not improve cholangiocyte growth, but induced polarization in cholangiocytes. TGF $\beta 1$ also induced polarization in cells in the presence of laminin in HA gels. In future, combined effect of jagged1 and TGF $\beta 1$ will be studied. Development of HA hydrogel culture system for liver cells would enable the construction of reproducible liver biliary organoids for drug discovery and transplantation.

\section{Adherens junctions and integrins cooperate during Drosophila embryonic wound closure}

\section{R. Hawkins, C. Schimmer, K. Rothenberg, R. Fernandez-Gonzalez}

Institute of Biomedical Engineering, University of Toronto; Translational Biology and Engineering Program, Ted Rogers Centre for Heart Research; Division of Engineering Science, University of Toronto; Department of Cell and Systems Biology, University of Toronto; Faculty of Life Sciences, Heidelberg University

Embryos repair wounds rapidly, in a process driven by collective cell movements [1]. Adherens junctions, molecular structures that mediate cell-cell adhesion, play important roles in embryonic wound closure. Adherens junctions are removed from the wound edge and localize to discrete points around the wound (tricellular junctions, TCJs). Junctional rearrangements are necessary for rapid wound healing [2, 3]. Cells also adhere to the extracellular matrix (ECM) via integrins. Coordination of cell-cell and cell-ECM adhesions is necessary for collective cell movements [4]. However, the role of cell-ECM adhesion in embryonic wound closure is unclear.

We investigated the interplay between cell-cell and cell-ECM adhesions during embryonic wound healing in Drosophila. We induced epidermal wounds by irradiation with an ultraviolet laser. Embryos expressed the adherens junction protein E-cadherin tagged with a red fluorescent protein. We tracked E-cadherin dynamics using confocal microscopy. To automate TCJ detection, we trained a support vector machine to identify regions containing TCJs, and we used maximum pixel intensities to extract TCJ positions from each region. We compared TCJ dynamics in controls and in embryos treated with RGDS, an inhibitor of integrin-ECM adhesion. RGDS treatment slowed wound closure. Before wounding, the initial E-cadherin intensity at TCJs was 35\% higher in RGDS-treated embryos, suggesting a compensatory effect. During wound closure, E-cadherin levels at TCJs in controls increased by $26 \% 40$ minutes post-wounding. In contrast, in RDGS-treated embryos, E-cadherin levels at TCJs decreased by $40 \%$. These results suggest that cell-ECM adhesion is required for E-cadherin accumulation at TCJs to drive rapid wound closure.

\section{The Metacognitive Processes by Which Active} Learning Promotes Problem-Solving Skills

\section{Sarvenaz Hosseini, Micah Stickel}

Department of Computer and Electrical Engineering, University of Toronto

Several researchers have found that problem-solving skills precede technical knowledge as necessary competencies in engineering occupations [1]. Simultaneously, other researchers have shown that active learning classrooms are more effective than traditional classrooms in fostering problem-solving skills [2]. However, researchers often do not uncover the mechanisms by which active learning classrooms cultivate such outcomes. Our team believes that by determining these mechanisms, we can better endorse active learning strategies to the engineering education community such that students can better prepare for engineering professions.

We are conducting an experiment for investigating the impact of active learning techniques in students' metacognition and the metacognitive processes by which students successfully solve problems. Metacognition is often characterized as an awareness of one's knowledge and the capacity to regulate the application of that knowledge for accomplishing tasks [3]. We chose metacognition as the mechanism linking active learning and problem-solving due to the argument that active learning makes learning "conscious and explicit", inciting students' metacognition [3].

We have developed our experiment methodology, which consists of holding workshops in the fall of 2020 for an experiment and control group. All students will be assigned problems from a first-year Statics course. The experiment group will solve the problems using active learning techniques. We will collect data about students' metacognition and 
problem-solving skills by conducting think-aloud assessments and administering surveys. We hypothesize that participating in active learning workshops will help students to improve upon their metacognitive knowledge and regulation. This way, students are able to successfully resolve problems.

\section{A Regular Expression Kernel for Matching and} Filtering Needs Ranging from Internet Exchange Points to Targeted Advertising Exchanges

\section{Truman Jian, Professor Hans-Arno Jacobsen}

Middleware Systems Research Group, Edward S. Rogers Sr. Department of Electrical and Computer Engineering

The establishment of The Internet of Things (IOT) in today's era has generated exponential amounts of data, demanding innovations in processing algorithms. One particular problem involves categorizing the input data through large databases of regular expressions, forming the basis of many filtering applications. They experience large volumes of expressions, frequent changing expressions, high input rates and high expressivity needs. One crucial area is rule and policy evaluation at internet exchange points to mitigate vulnerability exploits. Another critical application is identifying document tags for targeted advertising. Databases are expected to scale from hundreds of thousands to millions of expressions in the near future. Matching and filtering latency must be minimized to not impact traffic and user population. Due to the need for high expressivity, efficient matching is a challenge that requires optimized and thoughtful algorithm design.

Through this research, we are hoping to design and develop a novel algorithm that can fulfill these challenges. We are currently building an experimental framework to validate earlier designs to this problem and examining their limitations[1]. Furthermore, we are experimenting with state-of-the-art parallel computing technologies for further optimization.

The outcome of this research is poised to impact a wide variety of applications by minimizing the latency associated with matching and filtering in an age of rapidly growing data.

Extracting Key Features from EEG Signals to Classify the Attention Levels of Students

\section{Aoran Jiao, Crystal Liu, Scott Ramsay}

Division of Engineering Science, University of Toronto; Department of Materials Science and Engineering, University of Toronto

Attention is key to an effective learning experience. [1] [2] Traditional ways of assessing the attention levels of learners involve the instructors' observation of the physical expressions and feedback, which may not always be accurate and accessible with the increasing popularity and necessity of online learning. [3] Therefore, electroencephalography (EEG) signals are becoming more popular to determine attentiveness in recent research because they quantitatively and noninvasively reflect brain activities. [4] [5] [6] [7] However, these studies tend to produce diverging results based on different experimental designs with varying attention classification accuracy. To address this gap, the purpose of this research is to extract the key features from EEG signals based on results from previous literature and to develop a comprehensive classification algorithm to determine the attention levels of students.

The experimental procedure includes baseline data collection, signal preprocessing, feature extraction, and verification. Participants follow instructions to collect EEG data of purely focused and unfocused baseline activities using an off-the-shelf EEG headset with eight electrodes. Noise and artifacts are removed from the raw EEG data through an automated preprocessing pipeline. Features including alpha, beta, theta band powers, and the ratio of alpha power to beta power (Ra/b) are extracted using Fast Fourier Transform and power spectral density. Differentials between focused and unfocused features are analyzed statistically. Attention states predicted using these features are verified by participants to adjust the classification system and improve accuracy.

Preliminary results show that $\mathrm{Ra} / \mathrm{b}$ is higher in a focused state, corresponding to higher alpha power and lower beta power. Theta power does not show a distinguishing indication. Machine learning algorithms including the Support Vector Machine will be used when more data is collected to improve classification. The results will be increasingly relevant and applicable to educators and learners who are involved in an e-learning environment.

Investigating interventions to unlock student 
motivation in teams by strengthening selfefficacy

\section{Stephen Armen Joly, Emily Moore}

Institute of Leadership in Engineering Education and Deign, University of Toronto

There is an increasingly frequent demand from industry that the desirable skills engineering undergraduates should possess upon graduation is a teamwork-based skillset [1]. This has led to courses with a higher focus on team-based projects, however, many students report challenges with their teams where not all students are willing or capable to contribute equally to the project [2].

For institutions to develop students to be well-versed in teamwork dynamics, it is important to understand the relationship between positive teamwork experiences and educational structure in team-based design courses. The goal for faculty/staff should be to foster a pedagogical environment that effectively supports student teams, and that is conducive to better teamwork. We hypothesize this would significantly contribute to a classroom more conducive to positive team interaction.

This literature review aims to identify interventions led by instructors in Engineering Design, qualitatively assess their impact on self-efficacy, and understand the implications of each intervention on student motivation. Finally, an informal review of the University of Toronto first-year curriculum design courses, Engineering Strategies \& Practice (ESP) and Praxis, are included to determine which of the identified interventions have been employed by the Faculty.

In doing so, this paper will identify opportunities to implement new pedagogical interventions into the firstyear engineering curriculum that could improve the student experience in team design work by strengthening self-efficacy and teamwork motivation.

\section{User Interface For Oscillating Microfluidic Pump}

Parin Joshi, Liangcheng Henry Xu, Chun-Yu, Delphine Lin, Lidan You

Department of Mechanical and Industrial Engineering, University of Toronto, Toronto, Canada, Institute of Biomaterials and Biomedical Engineering, University of Toronto, Toronto, Canada
User interface design is the connection between the user and device. It comprises the design elements that need to exist for the user to be introduced to the device and allows the user to operate it. Previously, a microfluidic pump was built to generate an oscillating fluid flow using an Arduino microcontroller which can be utilized in modeling osteocyte mechanoregulation of breast cancer extravasation.[1] However, the existing user-interface for the pump only allows fluid flow shear stress input selection using a laptop which has made it difficult to use the pump as a miniature, standalone device. [2]

The present study investigates economical devices suitable to develop a compact, easy-to-use interface and an ArduinoIDE that would integrate the device with the pump to allow customisable inputs. After thorough investigation, various display devices were compared using a Pugh Chart and the compact, cost efficient and that allowed multiple inputs, 2.8"TFT TouchLCD, was suggested. The device could be directly attached to the microcontroller and doesn't require an external numpad for input which supported the compact design. The touch-screen library for ArduinoIDE was imported and a digital numpad was developed after understanding the coordinates of the device. The code for the interface was tested on Tinkercad, an Arduino simulator. Overall, this device enables multiple user input and allows the pump to function independently. The future steps include testing the Arduino code with the set up integrated with the microfluidic pump.

Relation between student perceptions and their performance in inquiry-based STEM education programs

\section{Sukhmani Khaira, Neal I. Callaghan, Locke} Davenport Huyer, Dawn Kilkenny

Institute of Biomedical Engineering, Faculty of Applied Science \& Engineering, University of Toronto.; Translational Biology and Engineering Program, Ted Rogers Centre for Heart Research, University of Toronto; Department of Chemical Engineering \& Applied Chemistry, Faculty of Applied Science \& Engineering, University of Toronto; Institute for Studies in Transdisciplinary Engineering Education \& Practice, Faculty of Applied Science \& Engineering, University of Toronto. 
Pursuit of post-secondary STEM education is rooted in direct interest in high school STEM as well as access to lab resources and academic support. Interest can be improved by engaging students in open-ended learning opportunities and/ or improving access to required STEM resources. Students from different schools across the range of aggregate cohort socioeconomic status show varied interest in the continued pursuit of STEM despite similar academic performance1; this leads us to wonder if their likelihood to persist in STEM education or careers may be based on perceived levels of access or self-efficacy rather than actual ability. At the University of Toronto, Discovery is an inquiry-based STEM education program engaging senior science students from local partner high schools of varied socio-economic status. This project examines differences in student attitudes towards the Discovery program, their individual performance in the classroom and their group performance in the Discovery program for partner schools.

Student data was collected in the form of regular individual classroom grades and Discovery group deliverable grades ( $n=2$ semesters). Qualitative data examining the student's underlying interest in STEM, level of preparedness for the final deliverable and perceived value of Discovery experience was collected using entrance and exit survey instruments. We expect to observe significant correlation between metrics of academic performance and attitudes towards STEM. We will also identify cohort effects determining how students who identify confidently with their STEM abilities will perform individually and in group.

In order to ensure that appropriate qualitative data is being collected to accurately capture student perceptions, it is imperative that survey questions are appropriately validated. Current study outcomes will define relevant educational trends and will aid the process of creating strong validated surveys for future Discovery programming.

\section{Geospatial Analysis on Metals and Polycyclic} Aromatic Hydrocarbons in Indoor House Dust

\section{Nadiha Khan, Marcia Pedroza Brambila, Arthur Chan}

Department of Chemical Engineering, University of Toronto

Indoor air quality is greatly affected by pollutants such as indoor house dust, which acts as a carrier of inorganic and organic pollutants including heavy metals and polycyclic aromatic hydrocarbons (PAHs), some of which are associated with outdoor sources [1][2]. Insight into the influences of geospatial factors including wildfire-related effects on these pollutant levels can impact health-based policies, understanding of transport of pollutants from their source, and wildfire remediation efforts. The purpose of this study is to assess whether the variability of metal and PAH levels found in the indoor house dust of homes is accounted for by geospatial factors. Concentration levels of 26 metals and 16 PAHs were measured previously using Inductively Coupled Plasma Mass Spectrometry and Gas Chromatography Mass Spectrometry, respectively, from entranceway samples of indoor dust in several residential homes in Fort McMurray, Alberta 1 to 2 years after the region was affected by a wildfire in 2016. Using the geospatial analysis tool, ArcGIS, the proximity of the homes sampled to potential sources such as major roadways and an airport were determined. Statistical tools including simple and multiple linear regression were used to analyze trends in the levels of metals and PAHs with respect to geospatial factors. Metals including arsenic, aluminum and vanadium had statistically significantly higher levels in homes from fire-damaged neighborhoods. No PAH showed a notable trend. Future studies should consider seasonality and the combined effect of indoor sources to provide a more accurate association of geospatial factors to indoor house dust.

Leaky-Wave Apertures for Satellite Applications Dana Kokoska, Elham Baladi, Gursimran Sethi, Sean Hum

\section{Electrical and Computer Engineering, University of Toronto}

Upon establishing a communication link with an orbiting satellite, ground space station antennas must physically move to track the path of fast-moving satellites. This movement is demanding on the mechanical parts of the antenna and consumes a lot of time [1]. Electronic beam-steering can change the direction of the antenna's beam without physical movement. This can be achieved using antenna arrays which steer the beam by changing the phases and time delays of the array, making communication with satellites more efficient [2]. 
The purpose of this project is to develop an antenna array that will perform electronic beam-steering. A reconfigurable leaky-wave array has been designed by Gursimran Sethi (a previous UofT Master's student) to combine the reconfigurable abilities of a transmitarray, and the directive ability of a leaky-wave antenna [3]. The array consists of multiple unit cells which each differ in the length of their striplines. The differing lengths correspond to varying phase delays and hence will make each unit cell reconfigurable [3]. The array will have an operating frequency of $19.5 \mathrm{GHz}$ and will be able to steer a sharp pencil beam of $25 \mathrm{dBi}$ gain to any angle [3].

We will be pursuing prototypes of the array layout for three different pencil beam angles. Thorough full-wave simulations in HFSS (finite element method solver) will be conducted beforehand, and the cavity that encases the array will be designed and fabricated. Recent progress was made to import stripline models into HFSS in the layout formation, and the previously plotted phase responses were verified with MATLAB.

\section{Consideration of Spalling When Analyzing} the Behaviour of Reinforced Concrete Beams Subjected to Combined Torsion and Bending

Chris (Seoyoung) Kwon, Allan Kuan, Prof. Evan Bentz

Department of Civil and Mineral Engineering, University of Toronto

Torsion acting in combination with bending and other loading behaviour is a common occurrence in many reinforced concrete structural components, such perimeter beams in buildings, bridge girders and other members curved in space. When designing these members for torsion, it is traditionally assumed that the cover concrete (concrete between the embedded reinforcement and the outside surface) has spalled off, a phenomenon which has been observed during experiments of beams tested in pure torsion. However, spalling has not been consistently observed during experiments involving torsion in combination with other actions. Because the mechanism for spalling is not well understood, design methods for members subjected to mixed loading which assume spalling tend to be conservative, resulting in materials being used less efficiently.

This project aims to examine the issue of spalling in reinforced concrete members subjected to combined torsion and bending. A previously developed numerical model which assumes spalling [1] has been implemented and modified to relax the spalling assumption, using more advanced constitutive models to capture the response of the concrete instead. The performance of the modified model will be validated against a database of beams in the literature. Preliminary results based on a subset of these beams tested in pure torsion show that the modified approach results in similarly accurate predictions to those made assuming spalling, but with a smaller variance. Recommendations will be developed upon completion of the validation study to inform the design and numerical modelling practices.

\section{Multi-agent motion planning for autonomous drones via deep reinforcement learning}

Yewon Lee, Chih-Chun Chen, Hugh H. T. Liu

Division of Engineering Science, University of Toronto; University of Toronto Institute for Aerospace Studies (UTIAS); Centre for Aerial Robotics Research and Education (CARRE), University of Toronto

Multi-agent motion planning algorithms enable autonomous drones, or unmanned aerial vehicles (UAVs), to travel from position $A$ to $B$ while avoiding obstacles and other UAVs. Such algorithms allow UAVs to perform natural resource monitoring and search-and-rescue missions [1]. Recently, a hybrid motion planning algorithm of deep reinforcement learning (DRL) and forced-based motion planning (FMP) called DRL-FMP was developed. DRL-FMP is less prone to collisions than older learning-based motion planning algorithms in virtual simulations [2]. However, DRL-FMP's stability and performance are compromised when implemented on real UAVs, which are affected by aerodynamic downwash effects arising from nearby agents.

Literature suggests that modeling UAVs as a cylinder may mitigate aerodynamic downwash by forcing UAVs to be vertically distanced from each other [3]. We investigate whether DRL-FMP's performance on real UAVs can be improved by modeling UAVs as cylinders. We test this cylinderbased DRL-FMP against ORCA [4], a baseline motion-planning algorithm. For each algorithm, 12 test cases are simulated in a physics-based platform with increasing number of agents and random initial and final positions. Then, we calculate 
the average percentage of successful scenarios, collision cases, and stuck scenarios for each test case to assess the performance of the cylinder-based DRL-FMP against ORCA.

So far, we have fully developed the cylinder-based DRLFMP and are integrating it with the simulation platform. Based on literature research, we expect that DRL-FMP can outperform ORCA on real UAVs. The results will provide insight into improving DRL-FMP's performance on real UAVs and help develop

\section{Unlocking the innate immune system:}

\section{Advanced biomaterial strategies hold the key} for improved wound healing

\section{Chen Yu Li, Katrina Vizely, Serena Mandla, Milica Radisic}

Department of Chemical Engineering and Applied Chemistry, University of Toronto; Institute of Biomaterials and Biomedical Engineering, University of Toronto; Toronto General Research Institute, University Health Network

Wound healing is a highly complex and carefully orchestrated process, dependent on the timed interactions of various cells, cytokines and growth factors [1, 2]. However, delayed healing, as a result of aging or disease, reduces the quality of life of those affected, and creates a significant economic burden on healthcare systems worldwide [3, 4]. During wound healing, the innate immune system is a key member in all stages, from hemostasis to remodeling, with macrophages undergoing a shift from a pro-inflammatory to a pro-healing [4]. In recent years, we have seen growing interest in therapeutic biomaterials to promote wound healing in both acute and chronic lesions [5, 6]. Particularly, hydrogels and other matrices have gained significant attention, along with the incorporation of diverse bioactive molecules, such as peptides, nanoparticles, stem cells and growth factors $[1,7$, 8]. However, not enough consideration has been given to the potential immunomodulatory effects when designing these biomaterials, especially considering the importance of the immune system in wound healing. In this review, the most relevant biomaterial strategies for modulating the immune system during wound healing will be discussed, including mechanical properties, peptide/growth factor release, stem cell delivery and anti-inflammatory modulation. As the wounds require regulation, spatially and temporally, and the innate immune system is present throughout, a thorough investigation will be necessary. By the end of the review we will have emphasized the importance of recognizing the innate immune system when designing biomaterials to comprehensively tackle the dynamic wound healing process.

\section{EEG and Body Posture Based Multimodal Emotion Recognition}

\section{Chenqi Li, Dunja Matic, Hassaan Inayatali, Jonathan Chan}

Division of Engineering Science, University of Toronto; School of Information Technology, King Mongkut's University of Technology Thonburi

Emotion is an indispensable component of interpersonal interactions. Affective computing is necessary to smoothen human-computer-interactions. Emotion recognition not only makes computers more emotionally intelligent, but also prompts research on the nature of human emotions to inspire digital recognition techniques [1].

EEG is widely used for unimodal emotion recognition, with limited accuracy (60-80\%) [2]. A fusion of EEG with facial expressions and/or physiological signals (ECG, GSR...) are common multimodal approaches [3]. However, the invasiveness of physiological sensors and lack of facial expression data during the COVID-19 pandemic calls for a new multimodal approach.

This study aims to improve the current state-of-the-art detection accuracies by combining EEG with body posture analysis. While wearing an EEG device, subjects are presented with emotion eliciting pictures/videos from academic databases (OASIS, IAPS...). Participants' upper body reactions are recorded with a webcam, for analyzing body movements over time. To arrive on a consensus of the evoked emotion, the recorded videos are presented to multiple volunteers for crowdsourcing the most appropriate emotion labels. The chosen labels, EEG signals, body posture analysis form the dataset to train machine learning algorithms (CNN, RNN...) for emotion classification.

With limited data collected, no result is available. As literature has shown, body posture, like facial expression, is a major non-verbal communication of emotion [4]. Therefore, EEG and body posture are expected to provide features that complement each other, achieving higher detection accuracy 
than that of unimodal approaches. This approach is adaptable to the new norm of wearing face masks and social distancing, minimizing contact with physical sensors.

Beyond Black-Scholes: A Bayesian

Reinforcement Learning Approach in Option Pricing

\section{Xuanze Li, Amine Mohamed Aboussalah, Chi- Guhn Lee}

Division of Engineering Science, University of Toronto; Department of Mechanical and Industrial Engineering, University of Toronto

For years, option pricing has been regarded as a difficult problem, because the general solution usually involves solving high-dimensional Partial Differential Equations (PDEs) which are very complicated to solve in practice. Due to the volatile nature of the stock market, very few researchers have demonstrated promising results in applying Reinforcement Learning (RL) algorithms into Option Pricing. We therefore want to explore the cases where we can tackle the European Options using RL approaches to solve the high-dimensional PDEs under different regime dynamics such as the Merton Jump Diffusion Model [1] and the Heston Stochastic Volatility Model [2].

Based on the previous studies [3],[4], we proposed a datadriven approach, by means of a Bayesian Neural Network (BNN), to evaluate European Options with the aim of accurately pricing European options in the regime-switching financial dynamics. By implementing an RL-BNN and comparing the performance with previous solutions under the Merton Jump Diffusion Model, we have demonstrated that the RL-BNN solver can accurately price and hedge highdimensional European options. The design process of our BNN-RL framework consists of applying Bayes rules to not only learn a point estimate value of the options but also to generate confidence intervals which could be used in risk management. Our final solution meets our requirements: it is backed up by mathematically sound theory, is computationally tractable and outperforms the existing models.

Our next step would be to further explore the RL algorithm to let it both solve the Black-Schole PDE and the Quantum Schrodinger PDE in the European option pricing cases, because both quantum physics and finance are probabilistic fields. We will investigate solutions from the Schrodinger PDE equation on option pricing and then compare the RL efficiency for both PDE classes and the accuracy on the learned solution.

\section{Developing hyaluronan hydrogels for liver organoids culture}

\section{Tracy Liu, Muhammad Rizwan, Molly Shoichet Institute of Biomaterials and Biomedical Engineering, University of Toronto}

Due to donor liver shortages, bioengineered livers provide sustainable alternative to treat the enormous numbers of patients suffering from end-stage liver diseases. The current liver organoids are cultured in mouse tumor-derived, poorlydefined Matrigel $₫$ and with animal cells, thus unsuitable for clinical applications and long-term liver function. Shoichet lab developed hyaluronan (HA) based chemically defined hydrogel for liver organoids differentiation and culture without the need for animal cells. Viscoelasticity and stiffness of the extracellular microenvironment (ECM) affect 3D cell growth and self-assembly in gels. However, the effects of crosslinking degree and the composition of HA based gels on viscoelasticity and stiffness are not explored. We hypothesize that the HA concentration will affect the viscoelasticity of the gels, which will be further modulated by the inclusion of ECM proteins in the gels such as collagen and laminin - essential for cell-matrix interaction. In this project, we synthesize HA hydrogels crosslinked with oxime click chemistry and analyze the effect of crosslinking degree, amount of HA, collagen, and laminin on the viscoelasticity of the HAoxime gels using rheology and micromechanical testing. We then benchmark against the mechanical properties of the liver tissue. Finally, we culture liver cells in various gels and analyze the growth and organoids formation to study the effect of viscoelasticity and stiffness on hepatocyte functions by using PrestoBlue assay and image analysis. We expect that the gels with mechanical properties matching the liver tissue, will promote liver cells viability and organoids formation. The results of this project will help to improve hydrogel design for the generation of liver organoids.

Redesigning Plastic Degradation Proteins and SARS-Cov-2 Spike Protein Inhibitors: A Machine 


\section{Learning Approach}

Tianyu Lu, Ryan Zhang, Alston Lo, Isha Sharma, Samantha Unger, Rochelle Wang, Andrew Gritzevskiy, Esther Yoo

University of Toronto

Improving the functional capabilities of proteins is a fundamental task in biological engineering, and may play a role in solving grand challenges such as accelerating enzymatic plastic degradation or designing inhibitors for viral proteins. Contemporary approaches to protein design often employ directed evolution, wherein a screening process following mutagenesis filters proteins by a desired trait [1]. While this method has been successful in enhancing isolated functions of proteins, it is expensive both in time and resources [2]. Our process leverages machine learning to extract patterns from large protein datasets, enabling greater control over generated mutations while reducing the cost of experimentation. Our pipeline for the design of a polyethylene terephthalate (PET) degrading enzyme, PETase, uses a type of variational autoencoder, the D-TCVAE, capable of generating novel sequences while being interpretable [3]. A discriminative model, based on supervised learning, creates a meaningful representation of a given sequence [5]. From this representation, a shallow top model is trained to predict both catalytic activity and thermal stability of a given protein. We then combine the generative model and discriminative model with an algorithm called Conditioning by Adaptive Sampling (CbAS), forming a generalizable protein design pipeline that maximizes our protein's activity and stability [4]. Molecular dynamics simulations are used to verify the validity of the design pipeline. Finally, to generalize the pipeline to designing SARS-CoV2 spike protein inhibitors, we leverage recent advances in graph convolutional networks to incorporate information from structure [6]. Such a generalizable design pipeline will be able to find a protein sequence satisfying any desired biological function.

Continuous Nondestructive Testing for

Temperatures up to $600^{\circ} \mathrm{C}$ using Hightemperature Ultrasonic Transducers

Kimberley Orna, Jenna Del Fatti, Mina Torabi, Anthony Sinclair
Electrical and Computer Engineering Department, University of Toronto; Mechanical and Industrial Engineering Department, University of Toronto

Nondestructive testing (NDT) is used by industries to monitor equipment integrity. AMAG supplies industries NDT devices, including ultrasonic transducers that monitor high-temperature pipes. A piezoelectric crystal within these transducers converts between mechanical and electrical energy to transmit and receive ultrasonic waves, which are analyzed for defects [1]. Additional components provide damping and acoustic coupling for effective signal transmission.

Current high-temperature transducers deteriorate after $400^{\circ} \mathrm{C}$, thus minimizing lifespan [2]. As specified by AMAG, our goal is to design a $3 \mathrm{MHz}$ wideband ultrasonic transducer for continuous operation from $25-600^{\circ} \mathrm{C}$, while minimizing internal stresses to extend lifespan. The frequency requirements ensure good image resolution and flaw detection [2]. Research shows lithium niobate satisfies piezoelectric requirements at high temperatures, while fitting zirconia, alumina, and silver components inside a steel housing ensures proper wave transmission [2]. After researching temperature-dependent material properties, thermal stress simulations were run on COMSOL using finite element analysis.

Stress concentrations were found in the steel and alumina components, due to corners and different expansion rates. Adding fillets and small gaps minimized these stresses. The crystal exhibited stresses up to $2 \mathrm{GPa}$ due to restricted radial expansion. Further model and simulation adjustments are required to allow for expansion and more accurate results.

Once optimized, the transducer will be built and tested at various temperatures for its detection capabilities. Project completion will benefit industrial plants that use NDT for safety improvement. The continuous, long-term operation of these transducers at various temperatures offers earlier flaw detection, motivating more economical and sustainable practices.

Development of longitudinal tracking methodologies to assess the long-term impact of the Discovery STEM educational initiative Angelina Ouyang, Neal I. Callaghan, Locke 


\section{Davenport Huyer, Dawn Kilkenny}

Institute Biomedical Engineering, University of Toronto; Translational Biology and Engineering Program, Ted Rogers Centre for Research, University of Toronto; Department of Chemical Engineering \& Applied Chemistry, University of Toronto; Insitute for Studies in Transdisciplinary Engineering Education \& Practice, University of Toronto.

Current STEM educational strategies present a gap between secondary and post-secondary pedagogical approaches, driving the need to bridge this divide. Discovery is an educational initiative that offers a novel approach, aimed at delivering inquiry-focused learning experiences for high school students based on an engineering capstone format, fostering open-ended problem-solving and critical thinking skills.

Preliminary evidence from previous Discovery studies indicate an impact on student participant attitude during their high school learning. However, we have not yet investigated how this learning shift translates to post-secondary study or early-career success. To assess this claim, the current study focuses on a thorough review of the literature to inform the development of a longitudinal study of post-Discovery participants to understand program impact on future pursuits.

The goal of a future longitudinal assessment includes up to eight-years of tracking, from the conclusion of Discovery participation (Grade 11/12) through post-secondary pursuits and/or professional careers. The five main components of study design are (1) tracking strategies, (2) duration and frequency of study, (3) ethical considerations of consent, (4) metrics of impact and (5) systematic analysis of results.

A strategy is developed by which to implement future longitudinal participant assessment. This tracking is achieved by employing an ongoing systematic review of participant Linkedln profiles, administering bi-annual surveys, and conducting yearly detailed interviews. Data will be subsequently analyzed to extract key results and findings. These outcomes will present an improved understanding of this novel learning platform and inform best practices for scale-up as a pedagogical practice.

Improving cognitive assessment through the gamification of traditional psychological tasks

\section{and tests}

\section{Scott Oxholm, Mark Chignell}

Mechanical and Industrial Engineering, University of Toronto

The benefits of cognitive assessment include detecting neurological conditions and identifying unsuitable medical interventions that increase the risk of brain damage. Assessment of executive functions in the elderly is of particular interest because these executive functions are strongly implicated in the ability to maintain independence. Psychologists have developed tools to test the different functionalities within the brain. However, many of these were developed using paper and pencil tests or physical materials (e.g. the Wisconsin Card Sorting Test [1]) or are challenging tasks that do not have an element of fun to encourage people to perform them.

The research gap being addressed here is that as the global population ages, there is an increasingly urgent need for efficient and effective methods of cognitive assessment that can be used on a large scale [2]. There needs to be an easier and more motivating way to measure executive functions than current standard methods which are inconvenient to use, and thus infrequently used.

Our purpose is to design games with the intent of collecting executive function data; hence allowing for cognitive assessment. Our objective is to make cognitive assessment fun and easy to complete - enabling less expensive and more frequent cognitive assessment. We have given researchers access to our software internationally. In turn, we can measure our design's effectiveness through researcher feedback, and repeatable statistics within our games (played by young and old audiences). To ease future game development, we are in the process of rewriting our code in a new web framework (React) to increase its modularity. Additionally, we are increasing data privacy and organization with a SQL relational database. Finally, we are developing a researcher's workbench which enables direct manipulation of game parameters for custom gameplay.

Investigating the Catalytic Performance of Supported Vanadium Oxide Catalysts in the Partial Oxidation of Methanol Aytekin (Anthony) Ozguler, Yinrui Xu, William 


\section{Broomhead, Junfeng Guo, Ya-Huei (Cathy) Chin}

Department of Chemical Engineering and Applied Chemistry, University of Toronto

The catalytic partial oxidation of renewable biomassderived methanol is an important reaction in the synthesis of polyoxymethylene dimethyl ethers (OME), an additive to diesel fuels reducing soot formation during combustion [1]. Heterogeneous metal oxide catalysts are used in creating chemical fuels and environmental applications because of their valuable oxidative and acidic properties and the ease in separations compared to homogeneous alternatives [2]. This study investigates the selectivity of OME in the methanol and oxygen reaction network using a supported vanadium oxide catalyst. The active site, vanadium oxide, is supported on titanium oxide at a weight-loading of 10\% (V2O5-TiO2). The synergy between the active site and support determine the oxidative and acidic activity of the catalyst, and, consequently, the conversion and selectivity of methanol. The kinetically relevant $\mathrm{C}-\mathrm{H}$ cleavage of adsorbed methanol creates formaldehyde, which further reacts with methanol in an acid-catalyzed reaction, forming OME [3]. Undesired reactions can occur by further oxidizing formaldehyde, producing formic acid, $\mathrm{CO} 2$, and water, which then react with methanol creating dimethyl ether and methyl formate [4].

Reactions are done in an isothermal gas-phase quartz reactor and detected using a packed column gas chromatograph. Tests are done to determine the reaction rate's order to methanol and oxygen and the effect of gashourly-space-velocity on selectivity. Furthermore, the reaction is simulated using ASPEN Plus and MatLab to validate the thermochemical properties of the reaction mixture. Obtaining an understanding of the oxidation of methanol using heterogenous catalysts will help optimize processes converting renewably derived biomass stocks into fuel alternatives.

\section{Statistical Inference on the Effect of Additives on Neutral pH Polymer Electrolytes}

Marlon Palmer, Alvin Virya, Keryn Lian

Department of Materials Science \& Engineering, University of Toronto

Aqueous-based solid electrochemical capacitors enabled by polymer electrolytes are environmentally friendly and safe energy storage devices that can be charged and discharged quickly with long cycle life. They are suitable for powering hybrid vehicles, portable electronics, and Internet-of-Things devices [1]. Nonetheless, their energy densities are bounded by their narrow voltage window due to water decomposition in the electrolytes [2]. To improve this, polymer electrolytes with a wider voltage window from neutral $\mathrm{pH}$ ion conductors that are inexpensive, chemically inert, and high in ionic conductivity have been developed [3]. Among various polymer electrolytes, a system comprised of neutral pH Na2SO4 conductor and polyacrylamide (PAM) host has demonstrated very promising performance at ambient conditions [4]. However, their applications are limited at low temperatures.

In this study, anti-icing additives were incorporated into Na2SO4-PAM binary system to improve the low temperature performance while maintaining their good performance at ambient temperatures. This improvement will allow the usage and operation of electrochemical capacitors all yearround in Canada's subarctic climate. The electrochemical properties with additives were studied using electrochemical characterizations such as cyclic voltammetry and impedance spectroscopy. The influential effects were statistically analyzed using design of experiment (DoE) methodologies using Microsoft Excel and Minitab. Current results show that using additives has the effect of increasing capacitance and ionic conductivity at low temperature. The effects of additive structure on the properties still require statistical analysis.

\section{The determination of acid resistant commercial membranes for forward osmosis}

\section{Sunny Park, Noel Devaere, Vladimiros Papangelakis}

Department of Chemical Engineering and Applied Chemistry, University of Toronto

The mining and metals processing industries constitute a major portion of economic growth and job creation worldwide but are accompanied by large volumes of waste production. Mining effluents can be generally characterized as low $\mathrm{pH}$ solutions containing dissolved heavy metals, such as arsenic, and costly treatments are often required to neutralize the effluent and remove water from dissolved contaminants 
[1]. Forward osmosis is a novel technology with the capacity to separate mining and metals processing water with minimal energy requirements, but commercial membrane performance is limited by the neutral operational $\mathrm{pH}$.

Forward osmosis is a spontaneous process wherein a membrane selectively allows water to flow from a contaminated feed stream into a highly concentrated salt stream known as a draw. The now diluted draw solution goes to a secondary separation process where the water is recovered [2]. In acidic environments, forward osmosis membranes degrade as the polymers undergo acid hydrolysis [3]. Consequently, the purpose of this research is the determination of commercial membrane candidates resistant to acid degradation without pre-treatment of low $\mathrm{pH}$ feed.

Candidates must fulfill the following criteria: commercial availability of flat sheet modules, an operational pH less than 3 , and the ability to reject salt ions at greater than $90 \%$ rejection. Non-cellulose acetate thin film composite membranes show promise for greater steric hinderance against deacetylation mechanisms [2]. As a result, three commercial membrane candidates have been identified according to literature data and will undergo experimentation to evaluate their efficacy in mining and metals processing water removal.

\section{Evaluating the Impact and Influence of Socio- economic and Environmental Factors in Water and Sanitation Access: The Realities of Peri- Urban Communities is Mexico City}

\section{Adriana Díaz Lozano Patiño, Amy M. Bilton, Pablo Rivera Cotera}

Water and Energy Research Lab, University of Toronto

Water access and sanitation are key factors affecting quality of life. Mexico City has struggled to find effective solutions to a decades-long water crisis [5]. Informal settlements, referred to as peri-urban communities, showcase the profound effects this crisis has on the city's most vulnerable vicinities [6]. The complex hydrology and economic constraints on these neighborhoods have resulted in insufficient infrastructure, near daily water access issues, and ineffective sanitation systems $[4,7]$.

Numerous institutions have attempted to implement off-grid water and sanitation technologies since the early 2000s in peri-urban households [1-3]. However, challenges regarding water quality and system maintenance remain [1-2]. The need for a design framework that explores the local context of these communities is essential to provide sustainable solutions [1-3] and to identify where off-grid sanitation technologies would prove more effective than centralized-grid systems [3].

This study explores the correlation between socio-economic factors and access to safe water and sanitation systems in peri-urban Mexico. Regions where large water and sanitation projects have been completed were used to examine impacts and main factors influencing water and sanitation access. Data from Mexican databases and interviews performed by local NGOs, were analyzed via a logistic regression model. The results show significant differences in the usage of sanitation technologies based on the socio-economic characteristics of each peri-urban community.

Our findings will inform the development of a framework to evaluate future water and sanitation technologies. Future work includes close community engagement through site visits, individual interviews and gathering quantitative data regarding the use of water and sanitation systems.

An Image Analysis Workflow for Characterizing Adipocyte Lipid Droplet Expansion in a 3D in vitro Obesity Model

\section{Saifedine Rjaibi, Vera M. Pieters, Alison P. McGuigan}

Department of Chemical Engineering and Applied Chemistry, University of Toronto; Institute of Biomedical Engineering, University of Toronto

Worldwide obesity has tripled since 1975 , now affecting over 650 million adults [1]. Obesity is linked to several comorbidities including type 2 diabetes, cancer, and cardiovascular disease [2]. During obesity, excess caloric intake causes expansion and subsequent dysfunction of adipocytes-the lipid-storing and predominant cell type of fat tissue-leading to disease. To study adipocyte dysfunction, our group is developing a 3D in vitro obese-adipocyte model that recapitulates important obesity hallmarks, such as caloric overload. To mimic this property, the 3D model is stimulated with lipids to induce accumulation and expansion of lipid droplets in adipocytes, and subsequently imaged. However, the abundance of lipid 
droplets makes it challenging to count and measure their size upon imaging. Hence, this research aims to validate the 3D obesity model by developing an efficient and reproducible image analysis workflow for quantifying lipid droplets.

First, adipocytes are imaged using fluorescent confocal microscopy, resulting in a stack of 2D image slices. Stacks are transformed into single images by projecting their maximum pixel intensities. Using the FIJI software, a series of filtering, thresholding, and processing steps are optimized to distinguish lipid droplets from each other and from background signal. Lipid droplets in lipid-stimulated and non-stimulated adipocytes are compared; lipid-stimulated adipocytes are hypothesized to have fewer but larger lipid droplets [3].

A robust and reproducible image analysis workflow provides validation of our 3D obesity model's physiological relevance, setting a foundation for investigating novel therapies targeting lipid droplet expansion-induced adipocyte dysfunction to prevent obesity-associated disease.

\section{Investigating Deployment Mechanisms for CubeSat Meta-Surface Antennas}

\section{Iliya Shofman, Sean Victor Hum}

\section{Division of Engineering Science, University of Toronto;} Edward S. Rogers Sr. Dept. of Electrical \& Computer Engineering, University of Toronto

Advanced electromagnetic meta-surfaces (MTS) have shown potential for use as high-gain, reconfigurable antennas aboard satellites [1], [2]. MTS are a promising alternative for traditional satellite antennas because they occupy less volume during launch but can expand to a larger area in orbit and thus increase signal strength. These properties make MTS antennas highly attractive for constellations of internet and communication satellites with limited on-board space [3].

To enable implementation of large-area antenna designs, researchers have been developing deployment mechanisms, or means to transform the antenna from stowed to operational configuration in space [4]. This study proposes a deployment mechanism tailored for an MTS antenna on a $6 \mathrm{U}$ CubeSat [5].

Industry sponsors expressed desire for a simple, reliable, and adaptable design. Therefore, the deployment mechanism with fewer parts, easier assembly, and higher degree of compatibility with commercially available off-the-shelf products was selected for further prototyping and testing.

A novel, versatile measurement apparatus was developed to enable accurate characterization of the deployed MTS system. Relative angles and position between target locations on the deployed systems will be measured photometrically, with smaller deviations from intended values indicating greater reliability. Mechanical deployment characteristics, such as oscillations and acceleration of components, will be assessed and mitigated to enhance reliability. Accuracy of the MTS scattering pattern, and its correlation with positional deviations, will be evaluated at the Reconfigurable Antenna Laboratory.

With higher-fidelity prototyping and fully integrated testing in progress, this study will enable the development and testing of more elaborate deployment mechanisms and advanced meta-surface antenna systems.

\section{Multi-agent motion planning for autonomous drones via deep reinforcement learning}

Yewon Lee, Chih-Chun Chen, Hugh H. T. Liu

Division of Engineering Science, University of Toronto; University of Toronto Institute for Aerospace Studies (UTIAS); Centre for Aerial Robotics Research and Education (CARRE), University of Toronto

Multi-agent motion planning algorithms enable autonomous drones, or unmanned aerial vehicles (UAVs), to travel from position $A$ to $B$ while avoiding obstacles and other UAVs. Such algorithms allow UAVs to perform natural resource monitoring and search-and-rescue missions [1]. Recently, a hybrid motion planning algorithm of deep reinforcement learning (DRL) and forced-based motion planning (FMP) called DRL-FMP was developed. DRL-FMP is less prone to collisions than older learning-based motion planning algorithms in virtual simulations [2]. However, DRL-FMP's stability and performance are compromised when implemented on real UAVs, which are affected by aerodynamic downwash effects arising from nearby agents.

Literature suggests that modeling UAVs as a cylinder may mitigate aerodynamic downwash by forcing UAVs to be vertically distanced from each other [3]. We investigate whether DRL-FMP's performance on real UAVs can be improved by modeling UAVs as cylinders. We test this cylinder- 
based DRL-FMP against ORCA [4], a baseline motion-planning algorithm. For each algorithm, 12 test cases are simulated in a physics-based platform with increasing number of agents and random initial and final positions. Then, we calculate the average percentage of successful scenarios, collision cases, and stuck scenarios for each test case to assess the performance of the cylinder-based DRL-FMP against ORCA.

So far, we have fully developed the cylinder-based DRLFMP and are integrating it with the simulation platform. Based on literature research, we expect that DRL-FMP can outperform ORCA on real UAVs. The results will provide insight into improving DRL-FMP's performance on real UAVs and help develop

\section{The Design of an Anti-Aliasing Filter for Thermal Monitoring of the SuperBIT Telescope}

\section{Kelly Werker Smith, John Hartley, C. Barth Netterfield}

Department of Electrical and Computer Engineering, University of Toronto; Department of Physics, University of Toronto; Department of Astronomy and Astrophysics, University of Toronto

The balloon-borne telescope SuperBIT measures the distribution of dark matter around galaxy clusters using weak gravitational lensing. Gravitational lensing, the bending of light due to the presence of mass, distorts the shapes of observed galaxies. However, systematic errors caused by vibrations or thermal variances can induce confounding aberrations in the galaxy shapes that SuperBIT observes [1]. Thermal variances are regulated using feedback from thermistors [2]. Aliasing during digital sampling and reconstruction leads to noisy thermistor signals, which constrains the precision of the temperature readings. Greater precision in the readings would support dynamic in-flight reactions to changes in temperature [3].

The purpose of this design is to increase the precision of the temperature signal readouts from $\sim 0.1$ Kelvin to $\sim 0.001$ Kelvin by way of a compact, stiff, $50 \mathrm{~Hz}$ stop-band anti-aliasing filter card. The $50 \mathrm{~Hz}$ cut-off frequency ensures that aliasing does not occur since it is below the $250 \mathrm{~Hz}$ Nyquist frequency. A compact design, measured by card area, allows for versatile spatial arrangements. Multiple mounting points decrease the lengths between centres of support, which increases the card's stiffness and decreases vibrations.

After simulations of different filter configurations and component values, a second order Butterworth filter in the Sallen-Key configuration was selected for its frequency response. The filter is implemented on a printed circuit board mounted to an Arduino. Layout was completed with attention to compactness and sufficient mounting points. After manufacture and assembly, the temperature readout precision will be tested using a function generator as a noise source.

\section{A Base Station for Bidirectional Radiofrequency Communication and Electromagnetic Induction Charging of Neural Implant}

\section{Jake Sprenger, Gerard O'Leary, Roman Genov}

Edward S. Rogers Sr. Department of Electrical \& Computer Engineering, University of Toronto; Institute of Biomaterials and Biomedical Engineering, University of Toronto

At the Intelligent Sensory Microsystems Lab, research is being done to examine the use of implantable devices to treat brain disorders. The device is implanted to collect data on the brain's electrical signals and makes use of machine learning to detect unusual activity (such as seizures). Once detected electrical stimulation is used to regulate these events. The advantage of a permanent implanted neuromodulator is that the user can go about their daily life, without the fear of seizures.

Chronically implanted devices present several unique engineering challenges. First, the collected data needs to be transferred to an external computer for analysis and to train machine learning models. Second, any battery-operated device will eventually run out of power, thus creating a need for a non-invasive battery charging method.

This presentation will address the design of a base station, which is a fully wireless solution for powering and data transmission. The base station includes a printed circuit board (PCB) which combines a Bluetooth Low Energy (BLE) transceiver and a Wireless Power Consortium (WPC) 1.2 standard compliant wireless power transmitter. This presentation will elaborate on the development and design of each of the three major components of the base station - the radiofrequency communication, wireless charging, and Graphical User Interface (GUI). Current progress has 
led to PCBs being sent for manufacturing, and a functioning prototype using a commercially available Bluetooth Integrated Circuit (IC). The next step in the research project is to evaluate the performance of the PCB solution.

Modelling Temporal and Spatial Variations of Nitrogen Dioxide Trends to Describe the Relationship between NO2 Pollution and COVID-19 Related Closures

Chuan Hui Sun, Greg Evans, Cheol-Heon Jeong

Department of Chemical Engineering \& Applied Chemistry, University of Toronto; Southern Ontario Centre for Atmospheric Aerosol Research

COVID-19 related closures have provided unprecedented opportunities to understand how anthropogenic activity impacts air quality. Nevertheless, air quality is highly variable; an analysis of past pollution levels in a region is important for drawing conclusions on abnormal trends, which has yet to be commonly established in Canada. NO2 is a key marker of diesel traffic-related air pollution, which relates to anthropogenic activity [1]. This project will analyse NO2 levels in Canada by temporal and spatial variation, with the goal of providing supporting evidence for the relationship between current NO2 perturbances and COVID-19 related closures.

The analysis of temporal variations was pursued by modelling NO2 trends using a combination of meteorological variables at a specific location. The variables investigated were temperature, humidity, precipitation, wind speed, and solar irradiance, as they are related to the chemical and the physical removal of NO2 [1][2]. Redundancy in variables was reduced prior to modelling by analysis of collinearity. These individual models explain temporal variations of $\mathrm{NO2}$, while spatial variations were explored by comparing models for different locations.

Thus far, a statistically significant model was created to predict NO2 levels in Toronto, achieving an adjusted R-squared of 0.55 . This model can explain the "normal" dependence of $\mathrm{NO} 2$ on meteorological variables. Moving forward, a comparison with trends observed in 2020 will be used to validate the presence and magnitude of abnormal trends in NO2 during COVID-19 closures. Additionally, models for different locations across Canada will be analysed to highlight spatial variations.

Immobilising Nickel based Catalysts on Biocarbon to increase output in the Sabatier Reaction

\section{Purushoth Thavendran, Charles Q. Jia}

Department of Chemical Engineering and Applied Chemistry, University of Toronto; Green Technology Laboratory

The advancement of space explorations that require extraterrestrial human habitats needs a steady supply of oxygen retrieved from the electrolysis of water. The Sabatier reaction studied by NASA for many years recycles waste hydrogen from electrolysis and carbon dioxide from the environment into methane and water [1][2]. The methane is used for fuel and water is used to generate oxygen. This reaction happens in the presence of a nickel catalyst. Previous designs including nickel-alumina/silica complexes provide moderate conversion of carbon dioxide and selectivity towards methane [3].

This project aims to find biocarbon's effectiveness in this reaction as catalyst support. Biocarbon is the solid product from thermally decomposing biomass. Biocarbon's surface functional groups and highly porous structure can make it cost-effective and advantageous support [4]. The support will provide a surface where carbon dioxide and hydrogen react on the nickel interface to provide greater conversion efficiency of carbon dioxide and selection efficiency for methane. Modifying the porosity, surface functional groups, sonication time with aqueous nickel, steaming times, and activation of the biocarbon impact the mass transfer, adsorption, and kinetics in the system.

These samples will be tested in a tube reactor. The amount of water produced will be measured to determine selectivity and conversion under 6 seconds of dwell time in the catalyst. Through experiments, the catalyst bed will be further refined to determine the characteristics of the ideal nickel-biocarbon catalyst system for optimal production of water and the potential of Biochar as a catalyst support in a multitude of reactions.

COVID-19 Identification Based on Chest X-Ray 


\section{Images Using Convolutional Neural Networks}

\section{Maggie Wang, Amy Chen, Chenqi Li, Grace Wu, Jonathan Chan}

Division of Engineering Science, University of Toronto; School of Information Technology; King Mongkut's University of Technology Thonburi

The outbreak of the COVID-19 pandemic has led to tremendous stress on medical systems across the world [1]. Recent advances in deep learning have inspired the use of algorithms to classify COVID-19 chest x-ray images-as chest $x$-rays are accessible, non-invasive, and can be used repeatedly to monitor the evolution of the disease [2]. A major issue faced by current deep learning algorithms is the lack of available COVID-19 datasets, which varies in quality and is highly imbalanced, leading to overfitting and bias in algorithms [2].

To address this issue, we use a convolutional neural network to provide accurate COVID-19 classifications, which is designed and trained to mitigate overfitting. We use techniques such as data augmentation, regularizers, increased input image size, and dropout layers to improve the performance metrics. As accuracy does not always reflect a model's performance for largely imbalanced datasets, the sensitivity, specificity, and F1-score of the model's predictions on the validation data will also be used to evaluate the model's performance. Grad-CAM will be used to visualize areas the model uses to make predictions, to ensure that the model makes predictions based on the diagnostically relevant areas [3].

The model achieved an $\mathrm{F} 1$ score of $98 \%$ on a validation dataset of COVID-19 and no-findings images during initial evaluations. The final prediction may aid radiologists in accurately detecting the disease or prompt further diagnostic examinations by detecting findings that were initially overlooked. As an extension to this work, preprocessing techniques and a multiclass model can be developed to obtain better results

\section{Evaluating Performance of Double-Pumped Interconnect in Coarse-Grained Reconfigurable Arrays}

Xinyuan Wang, Tianyi Yu, Hsuan Hsiao, Jason H.

\section{Anderson}

Dept. of Electrical and Computer Engineering, University of Toronto

We consider double-pumped interconnects as a means of area reduction in coarse-grained reconfigurable arrays (CGRAs) to conserve chip resources. Mult-pumping is an approach where a sub-circuit is clocked at a multiple of the system clock frequency. With double-pumping, the multiple is 2. CGRAs are programmable hardware platforms with large ALU-style logic blocks and word-width programmable interconnect. While multi-pumping has been previously explored for resource reduction in field programmable gate arrays (FPGAs), a lack of related research has been conducted for CGRAs. Interconnect multiplexers comprise a considerable portion of CGRA area. In this work, we apply double-pumping to halve the word-width of the interconnect multiplexers with an objective of conserving the overall CGRA area while minimizing frequency costs. We operate the interconnect at twice the system clock frequency, and the top and bottom half-words of a value are communicated in the first and second half of a system clock cycle. We have considered three circuit-level approaches for double-pumping in the ADRES architecture, and have evaluated them in comparison with the baseline in different CGRA sizes with varied interconnect richness. Area and performance consequences of all design points are assessed through a $45 \mathrm{~nm}$ standard-cell ASIC implementation. Preliminary area and frequency numbers have been taken after RTL synthesis and place and route. At the time of this abstract, the highest area reduction is observed to be $4.6 \%$ observed at a cost of $10.7 \%$ frequency. Next steps include synthesizing and evaluating these metrics across other architectures and double-pumping approaches.

\section{Promoting psychological safety through a handbook for virtual student design teams}

Katherine Whelan, Julianne Attai, and Patricia Sheridan

Division of Engineering Science; Troost Institute for Leadership Education in Engineering; Institute for Studies in Transdisciplinary Engineering Education and Practice, University of Toronto

With the switch to entirely virtual teams due to COVID-19, 
we have seen how the shift has made teamwork in design courses more difficult for everyone. This is due to the loss of shared physical spaces, varying time zones and access to technology, degrees of "zoom fatigue" [1], the difficulty in engaging, and the inability to physically prototype with the resources that the university offers.

This change in teaming context has created a need to produce a streamlined way for students and course instructors to develop teamwork, and collaborative learning, in now virtual design courses. To aid both students and instructors we intend to create a handbook that provides guidance and best practices for teaming and collaborative learning in this new space.

Our research focus started as a broad literature review on psychological safety in teams, trust, and online interactions. Psychological safety is defined as "one's emotional ability to express oneself in a virtual community without fear of negative consequences in relation to well-being, self-image, and status."[2] It has a positive impact on both the team's learning behaviors and the team's performance [3]. It also enables risk taking behaviors which will increase class and team engagement.

Currently we are compiling and framing our research into the handbook's sections. The goal is that the handbook will draw attention to the available technology, the impacts of an individual's identity online, connecting and building relationships, online community engagement, and provide insight on ways to promote psychological safety in each of these contexts.

\section{Optimizing the Culture Media and Digestion Protocol for Tumor-Macrophages Co-Culture on TRACER for Improved Downstream Analysis}

\section{Ziting (Judy) Xia, Ileana Co, Alison McGuigan}

Department of Chemical Engineering and Applied Chemistry, University of Toronto; Institute of Biomedical Engineering, University of Toronto

Pancreatic ductal adenocarcinoma (PDAC) is a malignancy having the worst prognosis amongst all solid tumors, with a 5-year survival rate of only 7-8\% [1]. The interaction between PDAC tumor cells and macrophage immune cells in hypoxia emerges as a promising target for immunotherapy [2]. The McGuigan Lab has developed a PDAC-macrophage co- culture system using their 3D tumor model named TRACER, which recapitulates the hypoxic tumor microenvironment. However, early experiments revealed two problems: 1) the co-culture media lacks sufficient optimization, and 2) the digestion protocol to access cells in TRACER for downstream analysis resulted in low yield and viability.

The presentstudyaims to optimize both media and digestion conditions in order to properly probe PDAC-macrophage interactions and minimize variability in downstream analysis. In Phase I, 5 media conditions with varying mixing ratios of PDAC/macrophage growth media were evaluated based on tumor cell growth and phenotype. In Phase II, two less digestively harsh enzyme types (Collagenase IV and Liberase) [3][4] with three concentrations were investigated. Their impacts on yield and viability were reflected from live/dead cell fluorescence emission measured with a plate reader.

Preliminary results from Phase I revealed the 50/50 mixing ratio as the optimal co-culture media based on its most proliferative PDAC cell growth. Future work will be devoted to determining the optimal digestion condition. The findings of this study will enable downstream scRNA-seq analysis to identify PDAC-macrophage gene pairs as treatable targets while demonstrating TRACER as a de novo 3D in vitro model to establish a co-culture system.

\section{Development of an Aspen Plus Model as an Optimization Tool for the Production of Polyoxymethylene Dimethyl Ethers}

\section{Yinrui Xu, Aytekin (Anthony) Ozguler, William Broomhead, Junfeng Guo, Ya-Huei (Cathy) Chin}

Department of Chemical Engineering and Applied Chemistry, University of Toronto

Incomplete combustion of diesel fuel leads to soot formation, a contaminant which can cause multifarious respiratory diseases [1]. Polyoxymethylene dimethyl ethers (OMEs), with chemical formula $\mathrm{CH} 3 \mathrm{O}-(\mathrm{CH} 2 \mathrm{O}) n-\mathrm{CH} 3$, are promising diesel fuel substitutes/additives that reduce the ecological and physiological impact caused by soot [1]. The synthesis of OMEs begins with a reaction between renewable methanol and formaldehyde, derivatives of $\mathrm{CO} / \mathrm{H} 2$ feedstock generated from gasification of woody biomass. The industrial production of OMEs involves complex reaction network and considerable unstable intermediates [2], hindering the 
understanding of the process; optimization of the emerging process is currently infeasible due to a lack of comprehensive information on thermodynamic parameters. This study aims at developing a software-based simulation model through Aspen Plus ${ }^{\circledR}$ to estimate the missing thermodynamic parameters, predict the equilibrium composition of product in liquid phase, and study the effect of temperature, pressure, and feed composition on the product distribution. The incipient construction of the model requires determination of thermodynamic properties of all species, including properties of intermediates that cannot be measured, due to their instability, by implementing a combination of limited literature resources, Group Contribution Methods and extrapolation from available experimental data. The current study focuses on examining the reliability of simulation model. A case study on estimated parameters and calculated chemical composition from a set of varied reaction conditions (i.e. reactants feed ratio and reactor temperature) was compared with literature results, lab experimental results, and pure mathematical optimization, for which a reasonable concordance is expected for an equilibrium simulation before the model could be used under dynamic, time-related conditions. The results of this study offer not only insightful understanding of the nature of reaction networks but also a dependable technical tool on prediction and optimization of the industrial production process of OMEs.

\section{Understanding Engineering Students'} Perspectives on Effective Teaching Strategies in Emergency Online Teaching

\section{Yahui Yang, Jiarong(Gerry) Chen, Dr. Qin Liu, Juliette Sweeney}

Division of Engineering Science, University of Toronto; Institute for Studies in Transdisciplinary Engineering Education \& Practice (ISTEP), University of Toronto

Due to the COVID-19 pandemic, the University of Toronto transitioned to emergency online teaching in mid-March 2020, and students moved to remote learning in all their courses. In this unusual situation, many of the teaching tools and strategies used for "conventional" online teaching [1][2] [3] were no longer relevant. The purpose of our analysis is to address what strategies remained or became relevant, and to better understand students' perspectives on what teaching tools and strategies worked best for remote learning.

An online survey was conducted in May with engineering undergraduates at UofT to capture students' experiences with remote learning, and to inform future teaching and learning. For our analysis, we selected two of the open-ended survey questions and analyzed the data using both thematic analysis and Natural language processing (NLP) / Machine Learning (ML) methods. Manual coding and thematic analysis were completed on NVivo and Excel. We are currently using the bag-of-words model, in NLP and GloVe embeddings and Recurrent Neural Network (RNN) in ML, to build an automated text analysis model, which aims to exceed the $74 \%$ accuracy rate achieved in a previous study [4].

Manual coding showed that students favoured lecture recordings and synchronous teaching, and identified areas for improvement. Our analysis using NLP and ML methods will not only enrich the findings from thematic analysis, but also help to assess the usability of NLP / ML in engineering education research. The results could be used to provide guidance for instructors to improve teaching techniques for future remote learning.

\section{An Open Source Software Library for DC} Magnetic Field Calculation around Arbitrarily Shaped Current-Carrying Coils using Richardson Extrapolation Assisted Numerical Methods

\section{Mingde Yin, Amar Vutha}

Department of Physics, University of Toronto

Accurately controlling the DC magnetic field in atomic spectroscopy experiments is required to measure transition energies, due to the Zeeman Effect otherwise incurring systematic error, caused by magnetic fields changing the gap between energy levels. Existing solutions are either closed source and difficult to expand into new projects [1], or slow and limited in functionality.

The project aims to create a computationally fast, accurate, and modifiable software library for calculating magnetic fields around current-carrying coils, designed for a new spectroscopy experiment. Calculations must span a $15 \times 15 \times 30$ $\mathrm{cm}$ volume, with a volumetric resolution of $1 \mathrm{~cm}$, and accept 
coils with varying shape, orientation, and current density.

Calculations are done by converting the coil into a series of vertices, then producing an array of discrete points in space around the coil and evaluating the magnetic field at each point in the array using the Biot-Savart Law and numerical integration. Performance was improved using vectorized mesh grids, and Richardson Extrapolation - which improves numerical integration accuracy without requiring additional function evaluations at smaller step sizes. Currently, the library does not consider singularity effects for points close to the coils, and the integration process can still be accelerated further. Fortunately, it can be modified to address this in the future.

The library was used to design the 3-axis DC magnetic field correction armature in the above experiment, which will help it better more precisely measure the $2 \mathrm{~s}$ hyperfine interval [2] in hydrogen, testing the validity of quantum electrodynamics and potentially opening the door to new physics.

\section{Low-Cost Seismic Base-Isolation System for Light-Frame Timber Houses in North America}

\section{Marawan Zaki, Constantin Christopoulos}

Undergraduate Student, Department of Civil and Mineral Engineering, University of Toronto; Professor, Department of Civil and Mineral Engineering, University of Toronto

Light-frame buildings constitute a great portion of North America's building inventory with close to 9 million homes erected in seismically active zones.[1][2][3] While earthquakes pose a low risk of collapse for these structures, they can cause great economic repercussions due to damage and occupant displacement. The aggregated losses over thousands of households in an area can be very large, as evidenced by the 1994 Northridge earthquake which caused over $\$ 20$ billion in damage to residential properties.[4] The introduction of a seismic base-isolation system can reduce the energy transferred to the building, allowing structures to remain operational during and after seismic events, reducing the financial and social burden of such natural disasters. Thus, the aim of this study is to develop and test multiple costeffective base-isolation systems, including sliding, rolling, rocking, and elastomeric bearing isolators. Each system's parameters including device configuration, material, and diaphragm integration will be optimized to achieve a lowcost yet effective system. Subsequently the study shall recommend the most optimal system and put-forth a design methodology for practical use. The systems' efficacy shall be determined by comparing both the numerical and experimental results of multiple configurations of average North American buildings with and without base isolation when subjected to a design basis and maximum considered earthquake. The isolation systems shall be jointly judged based on their cost and performance including peak story drift ratios, residual story deformations, floor accelerations, and visually observed damage.

Measurement and thermodynamic modelling of the solubility of rare earth tributyl phosphate complexes in supercritical carbon dioxide

Jilai Zhang, Adrian Lambert, Gisele Azimi

Department of Chemical Engineering and Applied

Chemistry, University of Toronto; Department of Materials Science and Engineering, University of Toronto

The growing ubiquity of digital devices and advanced electrical components has caused a meteoric rise in demand for rare earth elements (REE). These elements have unique physiochemical properties; hence, they are widely utilized in many technologically advanced products. Supercritical fluid extraction (SCFE) with carbon dioxide (sc-CO2) bears the potential to revolutionize REE production by replacing conventional hydrometallurgical extraction methods, which suffer from greatly elevated pollution and expense by comparison. SCFE employs the gas-like diffusivity and liquidlike solvation power of $\mathrm{CO} 2$ which is an abundant, safe, and inexpensive alternative to traditional solvents. SCFE utilizes chelating agents such as tributylphosphate-nitric acid (TBPHNO3) and Sc-CO2 to dissolve and transport REE ions as a chelated complex. Recent work shows this technique is highly efficient for extracting REEs from both primary and secondary feedstocks. However, data is lacking on the solubility of REE-TBP-HNO3 complexes in Sc-CO2.

This study aims to elucidate the relationship between temperature, pressure, and the solubility of REE-TBP-HNO3 
complexes. Previous studies have verified the applicability of thermodynamic modelling in depicting the behavior of SCFE systems, with models deviating from experimental results within 2\% [1]. Currently, an experimental method has been developed wherein solubility measurements are obtained using a UV-vis spectrometer and high-pressure cell by dissolving synthetic complexes at varying temperature and pressure. This data is used to construct empirical models to predict the solubility of REE-TBP-HNO3 complexes in Sc-CO2. These models are anticipated to shed light on the chemistry of SCFE systems and allow for optimization and scale-up of future SCFE operations.

\section{Local Stiffness Measurement Within Embryonic Tissue Using Acoustic force}

\section{Kaiwen Zhang, Min Zhu, Yu Sun}

Department of Mechanical and Industrial Engineering, University of Toronto; Program in Developmental and Stem Cell Biology, The Hospital for Sick Children; Institute of Biomaterials and Biomedical Engineering, University of Toronto; Department of Electrical and Computer Engineering, University of Toronto

Morphogenesis is a fundamental process of organ shaping, through the investigation of which we aim to understand how cells initially migrate and differentiate, as well as how diseases progress [1]. A key parameter that influences morphogenesis is local tissue stiffness, which is traditionally measured in terms of strain by displacing local parts of the sample with externally applied force [2-4]. While current methods, such as atomic force microscopy and optical/magnetic tweezers, are in use, they are invasive in that irreversible modifications such as probing and slicing to the sample tissue are inevitable and overheating due to excessive energy dissipation may be of concern [4, 5].

The objective is to explore, with knowledge from mechanical principles and applied biology, a new approach that can noninvasively measure the local stiffness of embryonic-level tissue. Here we propose to use a combination of acoustic force produced by acoustic waves and light sheet microscopy. Acoustic waves are non-invasive, while the microscopy is used to trace the displacement of cell nuclei within a tissue sample.

Simulation was completed in May to determine the appropriate frequency of the acoustic waves to achieve desired spatial resolution. Currently, numerical values of several parameters including wave amplitude, wave attenuation, and tissue density are being determined using phantoms that mimic the behaviours of embryonic tissue. Further experiments will be carried out on embryonic mouse tissue starting September. Stiffness at various locations within the tissue would provide qualitative data that may be used to further investigate its contribution in morphogenesis.

\section{Kinetic Modelling of UV/chlorine Advanced Oxidation Process in Drinking Water Treatment}

\section{Bo Zhao, Tianyi Chen, Nathan Moore, and Ronald Hofmann}

Drinking Water Research Group, University of Toronto;

The existence of micropollutants is ubiquitous in aqueous environment including drinking water, which is a potential risk to public health. UV/chlorine is a novel process that is capable of degrading micropollutants efficiently and economically. However, many stakeholders including consultants and utilities are unfamiliar with UV/chlorine, especially with its performance and costs. Thus, a kinetic model simulating the reactions between micropollutants and compounds within the UV/chlorine process is useful to help stakeholders examine its performance under various conditions, especially in respect of micropollutant removal efficiency and chlorine consumption.

However, existing models that simulate the UV/chlorine process may not include all aspects of interest in UV/ chlorine for the application in drinking water treatment. For example, some models ignore the common constituents in this type of reaction process with bromide and nitrate [1]. Additionally, current models may not be available to most stakeholders who intend to consider UV/chlorine in drinking water treatment. Thus, a kinetic model in the practical scenario of drinking water treatment with UV/chlorine will be developed in MATLAB ${ }^{\circledR}$. Currently, the simulation of chlorine and carbonate speciation in water has been achieved, by numerically solving the kinetic differential equation of each species using an ODE solver in MATLAB®. As more reactions and kinetic parameters are found in literature, the model framework can be updated to simulate UV/chlorine more accurately [2]. Ultimately, the kinetic model will be more 
available to stakeholders as a tool to predict and evaluate the UV/chlorine's performance, making it a feasible option to degrade micropollutants in drinking water.

\section{An in vitro hydrogel scaffold co-culture of} lymphangioleiomyomatosis cells with immune cells for improved LAM disease modelling

Spencer Zhao, Amber Xue, Molly S. Shoichet

Institute of Biomaterials and Biomedical Engineering, University of Toronto; Department of Chemical Engineering and Applied Chemistry, University of Toronto; Department of Chemistry, University of Toronto

Lymphangioleiomyomatosis (LAM) is a fatal lung disease characterized by abnormal growth of smooth muscle cells (SMCs), and which has no valid in vivo model to study. With our previously robustly designed three-dimensional hydrogel scaffolds, we were able to design a model that enabled us to mimic LAM cell behavior in vitro.[1] We now propose to upgrade our current model to allow for multi-cellular culture, where we will gain key insights into both LAM disease and the influence of drugs on LAM cell behaviour. Macrophages are abundant in the LAM lung environment and it is likely they may interact with LAM cells.[2] We hypothesize that a partially physically crosslinked self-healing hydrogel will allow for stable co-culture of LAM cells with macrophages, and thus more accurately model lung tissue. Hydrogels are comprised of protease-degradable chemically-crosslinked hyaluronan and physically crosslinked methylcellulose. Macrophages are encapsulated within gels while LAM cells are seeded peripherally and allowed to invade. Gel formulations were found to allow encapsulation, remodelling, and migration for both cell types. Cytokine analysis and flow cytometry of co-cultured LAM cells suggest a secretion and phenotypic behaviour more similar to natural LAM cells than those in monoculture. The results represent an important advancement towards modelling the complex lung environment in vitro and highlight the importance of considering both extracellular matrix and cellular components of a disease environment. Future work will involve drug screening and discovery using this newly developed scaffold.
Designing an Affordable, Easy-to-use EEG for Measuring Student Engagement in Learning Environments

\section{Kevin Zhu, Crystal Liu, Scott Ramsay}

Division of Engineering Science, University of Toronto;

2Department of Materials Science and Engineering, University of Toronto

Electroencephalograms (EEGs) could be potentially useful in the field of educational research, as they present a nonintrusive, real-time method of measuring student engagement during classes. Studies involving EEGs have already found correlations between brain waves and attention, student engagement, and brain-to-brain synchrony [1] [2] [3] [4].

However, commercially available EEGs are either obstructively expensive, or do not offer the correct capabilities. For example, Muse and NeuroSky headsets do not have enough measurement channels [5] [6], while OpenBCl and Emotiv EEGs cost upwards of 700 USD per set [7] [8]. Thus, an easily manufacturable, user-friendly, and cost-effective EEG headset is needed.

Manufacturability is measured by the difficulty of procuring materials, production, and assembly, either in time elapsed or actions needed. Usability is measured by comfort, weight, volume, and set-up time. The number of applicable electrode configurations and repair-ability are also considered. The performance of the EEG is measured by the clarity of data and the amount of noise that requires rejection.

The EEGs are prototyped using a mixture of 3D printed parts and off-the-shelf components. Each prototype is tested, first on a part-by-part basis, then holistically, using measurement processes associated with each criterion, such as Likert scale questionnaires for comfort, and recording assembly and repair times. New CAD designs and assembly processes are then iteratively developed to improve the performance. The current prototype is an 500 CAD, lightweight, easily manufacturable system that uses elastics to ensure the proper placement and pressure of electrodes. Next steps include streamlining setup, increasing adjustability, starting data clarity tests, and further decreasing cost. 